ARTICLE

\title{
Horizontally acquired papGll-containing pathogenicity islands underlie the emergence of invasive uropathogenic Escherichia coli lineages
}

\author{
Michael Biggel (10 ${ }^{1 凶}$, Basil B. Xavier (10 1, James R. Johnson², Karen L. Nielsen (10 ${ }^{3}$, Niels Frimodt-Møller ${ }^{3}$, \\ Veerle Matheeussen (10 ${ }^{1,4}$, Herman Goossens ${ }^{1,4}$, Pieter Moons ${ }^{1,6}$ \& Sandra Van Puyvelde (1) 1,5,6四
}

Escherichia coli is the leading cause of urinary tract infection, one of the most common bacterial infections in humans. Despite this, a genomic perspective is lacking regarding the phylogenetic distribution of isolates associated with different clinical syndromes. Here, we present a large-scale phylogenomic analysis of a spatiotemporally and clinically diverse set of 907 E. coli isolates, including 722 uropathogenic E. coli (UPEC) isolates. A genome-wide association approach identifies the (P-fimbriae-encoding) papGll locus as the key feature distinguishing invasive UPEC, defined as isolates associated with severe UTI, i.e., kidney infection (pyelonephritis) or urinary-source bacteremia, from non-invasive UPEC, defined as isolates associated with asymptomatic bacteriuria or bladder infection (cystitis). Within the $E$. coli population, distinct invasive UPEC lineages emerged through repeated horizontal acquisition of diverse papGll-containing pathogenicity islands. Our findings elucidate the molecular determinants of severe UTI and have implications for the early detection of this pathogen.

\footnotetext{
${ }^{1}$ Laboratory of Medical Microbiology, Vaccine and Infectious Disease Institute, University of Antwerp, Antwerp, Belgium. ${ }^{2}$ Veterans Affairs Medical Center and University of Minnesota, Minneapolis, MN, USA. ${ }^{3}$ Department of Clinical Microbiology, Rigshospitalet, Copenhagen, Denmark. ${ }^{4}$ Laboratory of Clinical Microbiology, Antwerp University Hospital, Antwerp, Belgium. ${ }^{5}$ Cambridge Institute of Therapeutic Immunology and Infectious Disease (CITIID), Department of Medicine, University of Cambridge, Cambridge CB2 OSP, UK. ${ }^{6}$ These authors contributed equally: Pieter Moons, Sandra Van Puyvelde.

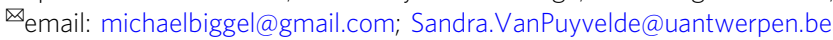


n humans, Escherichia coli is both a commensal and a pathogen capable of causing intestinal and extraintestinal disease. In the urinary tract, E. coli causes a wide range of clinical syndromes that includes, from less to more severe, asymptomatic bacteriuria (ABU), bladder infection (cystitis), kidney infection (pyelonephritis), and urinary-source bacteremia. An estimated half of all $E$. coli bloodstream infections originate from the urinary $\operatorname{tract}^{1-3}$.

E. coli strains with a special ability to cause urinary-tract infection (UTI) are classified as uropathogenic E. coli (UPEC). UPEC has further been grouped with $E$. coli strains that cause (non-urinary-source) bacteremia, meningitis, and prostatitis as extraintestinal pathogenic E. coli (ExPEC) ${ }^{4}$. UPEC are considered to be opportunistic pathogens, with the human gut as their reservoir ${ }^{5}$. The different pathotypes of intestinal pathogenic $E$. coli (enteropathogenic E. coli [EPEC], enterohemorrhagic E. coli [EHEC], enterotoxigenic E. coli [ETEC], enteroinvasive E. coli [EIEC], and enteroaggregative E. coli [EAEC]) are differentiated genotypically by their characteristic virulence genes. In contrast, E. coli isolates are often classified presumptively as ExPEC and/or UPEC according to their site of infection and isolation, irrespective of their intrinsic virulence, which usually is unknown ${ }^{6,7}$.

Although multiple urovirulence factors and their importance in UTI pathogenesis have been described, genotypical features that distinguish UPEC from non-pathogenic E. coli remain incompletely defined ${ }^{5}$. The identification of defining UPEC features is hampered by the large number of potentially redundant bacterial virulence-associated genes (VAGs), a shared habitat with commensal $E$. coli in the intestinal microbiota, and varying host susceptibility to $\mathrm{UTI}^{8}$. UTI pathogenesis is hence assumed to be determined by a complex interplay of bacterial and host factors.

The E. coli population is subdivided into phylogenetic groups (phylogroups) A, B1, B2, C, D, F, and G, and cryptic clades, with ExPEC and UPEC strains deriving predominantly from phylogroups B2 and D. Strains from phylogroups B2 and D typically have more VAGs than do those from phylogroups $\mathrm{A}$ and $\mathrm{B} 1$, which are associated with commensal and intestinal pathogenic $E$. coli ${ }^{4,9,10}$. Phylogroups B2 and D encompass the pandemic UPEC lineages clonal complex (CC) 69, CC73, CC95, and CC131, which are responsible for most cases of $E$. coli cystitis, pyelonephritis, and bloodstream infection worldwide. This predominance in extraintestinal E. coli infections of just a few E. coli lineages, among the hundreds that exist, suggests that specific genetic determinants facilitate the expansion, global spread, and virulence of these pathogens ${ }^{11}$. Increased antimicrobial resistance (AMR) might have contributed to the recent dissemination of CC69 and CC131, whereas it cannot explain the epidemiologic success of the largely susceptible lineages CC73 and CC9512-14.

Previous phylogenomic analyses provided important insights into the population structure, virulence associations, and AMR of ExPEC and UPEC ${ }^{12,13,15-18}$. However, a specific genomic analysis of invasive UPEC strains-defined here as those associated with severe UTI (pyelonephritis or urinary-source bacteremia)-is lacking. PCR-based studies targeting subsets of VAGs have identified a greater frequency of various VAGs in pyelonephritis and urinary-source bacteremia isolates, as compared to $\mathrm{ABU}$, cystitis, or fecal isolates ${ }^{19-22}$. These VAGs include papGII, which encodes one of several PapG tip adhesin variants of $\mathrm{P}$ fimbriae. PapGII binds to the globoseries of glycosphingolipids on uroepithelial cells and transcriptionally regulates host gene expression in kidney cells, leading to a pyelonephritis-associated IRF-7 response $^{23}$. Like many other E. coli VAGs, the pap operon encoding $\mathrm{P}$ fimbriae lies on pathogenicity islands (PAIs) ${ }^{24}$. PAIs are large horizontally transferable genetic elements assumed to play an important role in the evolution of pathogenic E. coli ${ }^{25}$.
In this study, we used a genomics approach to investigate the population structure, virulence determinants, and evolution of invasive UPEC isolates, as compared to non-invasive UPEC isolates, which were defined here as those associated with asymptomatic bacteriuria (ABU) or cystitis. Fecal isolates not associated with disease were included to investigate their genetic similarity with UPEC. Our analysis identified a small number of enduring and broadly distributed invasive UPEC lineages that seemingly emerged after independent horizontal acquisitions of papGII.

\section{Results}

Invasive UPEC cluster in distinct phylogenetic lineages. We analyzed whole-genome sequences of 722 total UPEC isolatesincluding 385 invasive UPEC isolates (from five collections) and 337 non-invasive UPEC isolates (from nine collections) - and 185 fecal isolates (from two collections). The 722 UPEC isolates were so defined based on their source of isolation and associated clinical presentation, whereas the fecal isolates were not associated with UTI or intestinal infection (Supplementary Note 1 gives the various source collections' inclusion criteria). The 16 isolate collections had a broad spatiotemporal distribution: they derived from various locales in Europe and the United States and spanned nearly four decades (1981-2018) (Supplementary Fig. 1, Table 1). For four of these collections (LtABU, MC_pye, MVAST_ABU, UZA_uro), the isolates $(n=151)$ were sequenced within this study; for the remaining 12 collections ( $n=756$ isolates) the sequence data were publicly available (Supplementary Table 1).

Phylogenetic analysis revealed a non-random phylogenetic distribution of the three clinical phenotypes, i.e., invasive UPEC, non-invasive UPEC, and fecal isolates. Invasive and non-invasive UPEC isolates alike were predominantly $(86 \%$ and $71 \%$, respectively) from phylogroups $\mathrm{B} 2$ and $\mathrm{D}$; by contrast, fecal isolates were more evenly distributed among phylogroups A, B1, B2, and D (Supplementary Table 2, Supplementary Note 2). Among the invasive UPEC isolates the 11 dominant CCs were CC12, CC14, CC23, CC31, CC62, CC69, CC73, CC95, CC131, CC144, and CC405 (the corresponding sequence types (STs) are shown in Fig. 1). These 11 dominant CCs accounted collectively for $82 \%$ of all invasive UPEC isolates, with limited variation between collections (Supplementary Fig. 2). Moreover, within these CCs, invasive and non-invasive UPEC isolates tended to cluster into distinct sublineages (Fig. 1). Most of these CCs encompassed one or multiple sublineages that were significantly enriched with invasive UPEC isolates $(P<0.05$, for proportion of invasive UPEC isolates within vs. outside the sublineage; Supplementary Data 2), giving 12 sublineages associated with invasiveness. Within these 12 sublineages combined, $86 \%$ of all UPEC isolates were invasiveness-associated, vs. $39 \%$ of other UPEC isolates.

Invasive UPEC lineages emerge after acquisition of papGII+ PAIs. A pan-genome-wide association study (pan-GWAS) comparing invasive vs. non-invasive UPEC isolates that included 30,705 clusters of orthologous genes (COGs) identified significant associations with invasiveness for two genomic loci, papGII and $i u c$, which encodes aerobactin biosynthesis (Fig. 2, Table 2). An alternative, gene-prediction-independent GWAS approach based on De Bruijn graphs and linear mixed models (DBGWAS ${ }^{26}$ ) confirmed papGII as the most significantly invasivenessassociated genomic region (Supplementary Table 3, Supplementary Data 3). Overall, papGII was present in $63.9 \%$ of invasive UPEC isolates, as compared with $15.4 \%$ of non-invasive UPEC isolates $\left(\mathrm{OR}=9.7, P=6.0 \times 10^{-42}\right)$ and, for reference, $19 \%$ of fecal isolates $\left(\mathrm{OR}=7.6, P=9.1 \times 10^{-25}\right)$. 


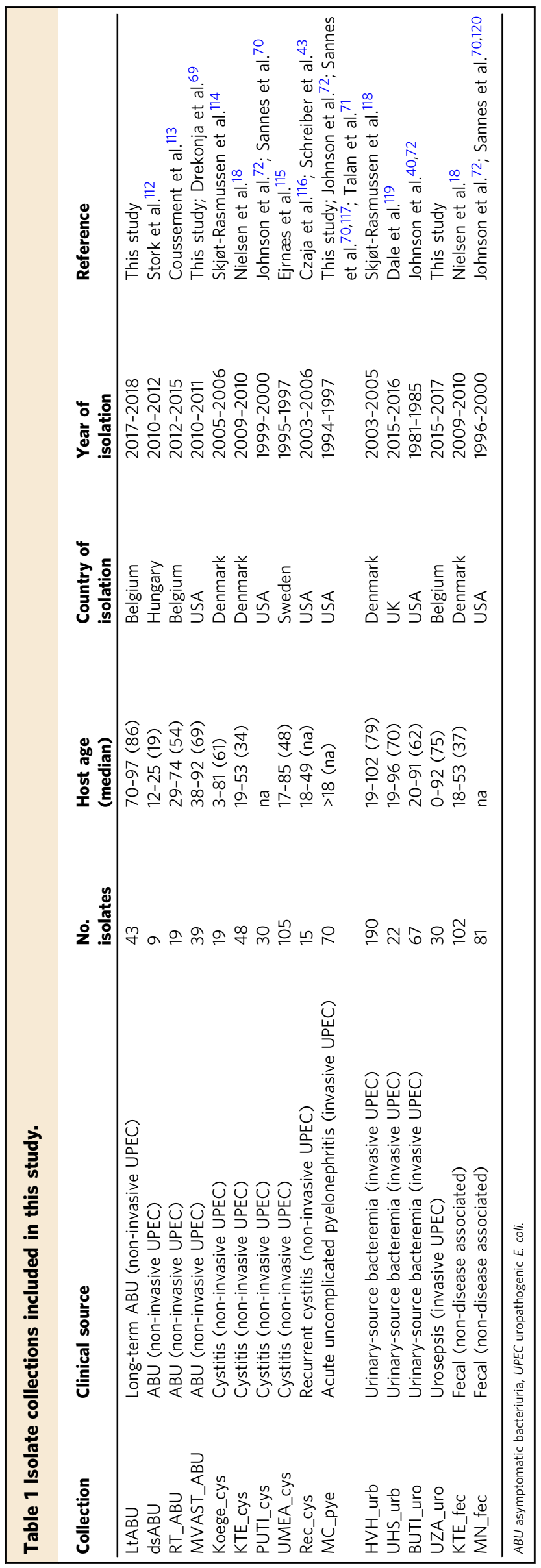

This significant association of papGII with invasive UPEC was found irrespective of host gender or age (Supplementary Table 4). In addition, within each clinical phenotype (invasive UPEC, noninvasive UPEC, and fecal) the frequency of papGII varied only modestly between collections, ranging by collection from 58 to $77 \%$ for invasive UPEC, vs. from 0 to $29 \%$ for non-invasive UPEC, and 19 to $20 \%$ for fecal isolates; the observed modest variation likely reflected the collections' differing settings and patient inclusion criteria (Supplementary Fig. 3). The presence of papGII ( $n=333$ isolates) coincided closely with the abovedefined invasive UPEC-enriched sublineages (Fig. 1); $88 \%$ of isolates in these sublineages carried papGII, vs. $19 \%$ of other isolates $(P<0.001)$.

The 333 papGII-positive ( $p a p G I I+)$ isolates clustered robustly in 14 discrete lineages, which thus were termed papGII+ lineages (Fig. 3a). CC12, CC14, CC31, CC59, CC62, CC69, CC95, CC131, CC144, and CC405 encompassed one such papGII+ sublineage each. CC73 showed a heterogenous population structure that encompassed four distinct papGII+ lineages (called L1-L4), as identified by using a second hierarchic level of clustering. Most papGII+ lineages comprised isolates from different regions that had been collected during different time intervals over 40 years, suggesting enduring, broadly distributed lineages rather than transient and/or geographically restricted subpopulations (Supplementary Fig. 2).

The genetic context of papGII in the 14 papGII+ lineages was usually unclear from short-read sequencing data only, but could be resolved for 35 isolates, including at least one from each papGII+ lineage. These 35 genomes comprised 14 publicly available, high-quality assemblies and 21 complete or nearcomplete assemblies obtained in-house using long-read sequencing (Supplementary Data 5). Six of the 35 genomes harbored more than one papGII locus. The total of 42 papGII genes were each part of a complete pap operon (papGII operons) that consisted of 11 genes (papIBAHCDJKEFG), with sequence variation found for $p a p A$ (major fimbrial subunit) and $p a p E$ (minor fimbrial subunit) (Supplementary Fig. 4) as described previously ${ }^{27,28}$. The papGII genes always occurred on PAIs, i.e., genomic regions flanked by an integrase, integrated into the chromosome at one of six specific loci, and absent in phylogenetically related isolates.

These 42 resolved papGII-containing (i.e., papGII+) PAIs were integrated directly downstream of the tRNA genes pheV, pheU, selC, or leuX, or into the gln or ula operons (Supplementary Fig. 5). In addition, the papGII+ PAI insertion site could be determined in 170 other papGII+ isolates (171 putative PAIs) for which only short-read assemblies were available. Overall, the tRNA-pheV and tRNA-pheU sites accounted for 194 (91\%) of all 213 identified papGII+ PAI insertion sites; the remaining 9\% were divided between four other tRNA sites, which accounted for $0.5-4 \%$ of the PAIs each (Supplementary Data 7). PAIs inserted at the (predominant) tRNA-pheV and tRNA-pheU sites shared the same integrase gene, whereas those inserted at the other four sites had distinct insertion-site-specific integrase sequences (Supplementary Fig. 6). Complete or near-complete assemblies of three papGII+ isolates each from CC144 and CC12 revealed the sporadic presence of two or three papGII operons, each on a distinct PAI (Supplementary Fig. 7).

The 42 resolved papGII+ PAIs showed a highly diverse gene content: the only consistently present element was the papGII operon (Fig. 3b, Supplementary Fig. 8a). This is in line with the concept that PAIs are organized in mosaic-like structures, consisting of a flexible pool of gene modules ${ }^{29,30}$. The papGII+ PAIs ranged in size from $28 \mathrm{~kb}$ (26 genes) to $146 \mathrm{~kb}$ (140 genes). They contained from 12 to 31 VAGs each (including the 11 pap genes), which encoded up to seven virulence factors per PAI 


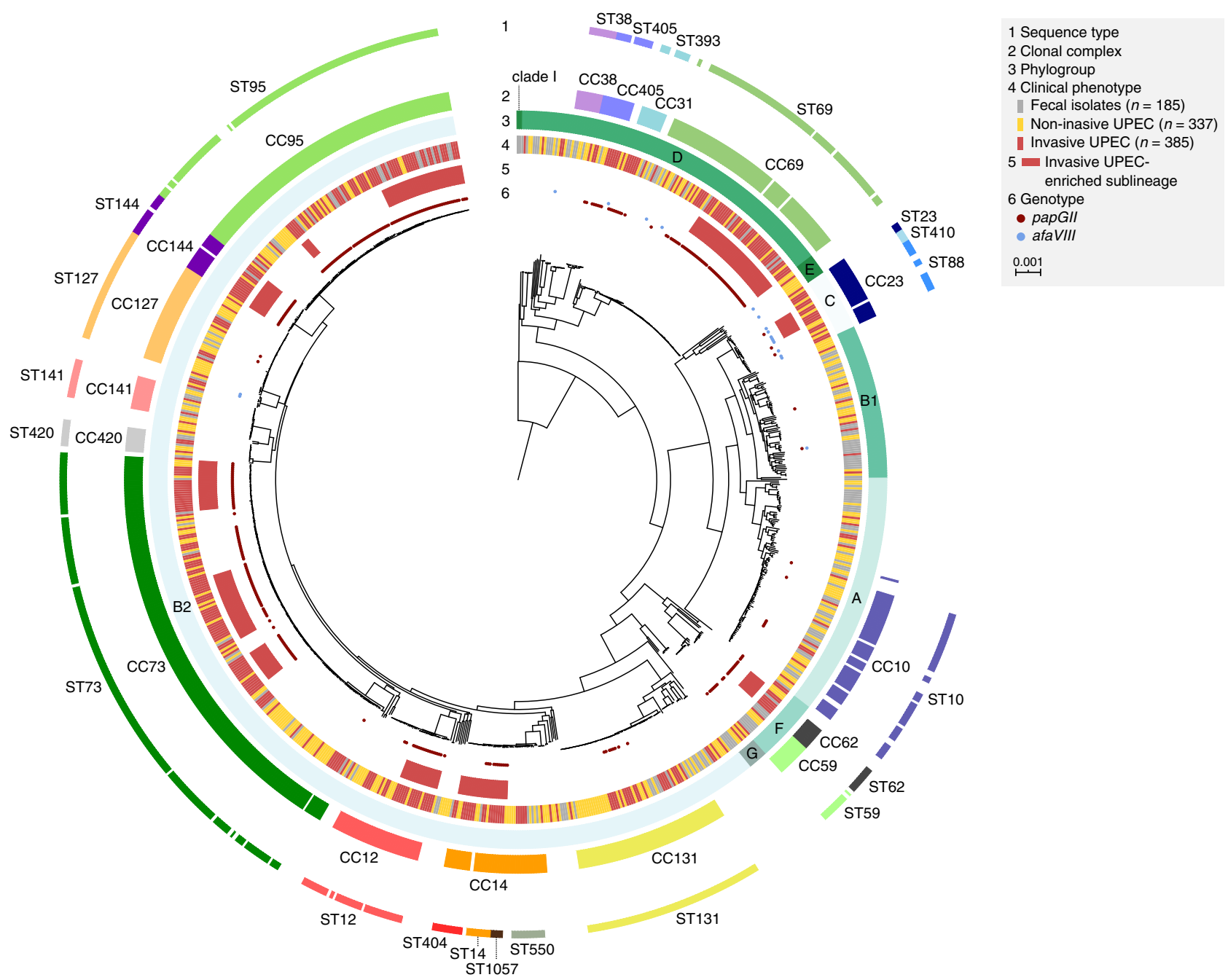

Fig. 1 Phylogeny of 907 Escherichia coli isolates associated with different clinical phenotypes. Midpoint-rooted maximum-likelihood phylogenetic tree based on 109,023 variable sites identified in a core genome alignment (1.136 Mbp). Ring 1, 2, and 3 denote predominant sequence types (ST), corresponding clonal complexes (CC), and phylogroup assignment. Clinical phenotypes are labeled according to the key (ring 4). Phylogenetic clusters identified using BAPS (Bayesian analysis of population structure) significantly enriched with invasive UPEC isolates are highlighted in ring 5. The presence of the afaVIII and papGII (blue and red dots) across the phylogeny is annotated in ring 6 . The scale bar indicates the number of substitutions per site in the core genome alignment. A tree with bootstrap support values is provided in Supplementary Fig. 20. The tree was visualized using iTOL 89 . An interactive visualization of this phylogenetic tree can be found out at https://microreact.org/project/O4QAYAJWw.

(Supplementary Fig. 8a, Supplementary Table 5). They also contained multiple hypothetical genes, phage gene remnants, and IS elements, but no AMR genes. Based on sequence similarity, the 42 resolved papGII+ PAIs could be grouped into six types, I through VI (Supplementary Fig. 8b). The incompletely resolved papGII+ PAIs of 199 additional isolates could be assigned to these six types by mapping reads to the resolved PAIs (Supplementary Data 11). Within a given papGII+ lineage, most papGII+ PAIs were fully conserved or differed only by a few IS elements, suggesting a single PAI acquisition event per papGII+ lineage (Fig. 3a). By contrast, the sporadic occurrence of similar PAIs in distantly related isolates suggested horizontal gene transfer events (Supplementary Fig. 9).

Exceptionally, one invasive UPEC-enriched sublineage was not characterized by the presence of papGII: although phylogroup C (represented almost entirely by CC23 isolates: 24/25, 96\%) comprised mainly invasive UPEC isolates $(17 / 25,68 \%)$, only three CC23 isolates contained papGII. Screening of CC23 isolates for known $E$. coli VAGs identified the afaVIII operon (afaABCDE-VIII) in eight (of 14) papGII-negative invasive UPEC isolates (Fig. 1). afa genes encode afimbrial structures that mediate adhesion and invasion ${ }^{31}$; some of these, including AfaEVIII, have been associated with pyelonephritis ${ }^{32,33}$. Over the entire dataset, afaVIII occurred in 15 invasive UPEC isolates $(3.9 \%)$, as compared with only two non-invasive UPEC isolates $(0.6 \%$; OR $=6.8, P=0.003)$ and one fecal isolate $(0.5 \%: \mathrm{OR}=7.4$, $P=0.03)$. These afaVIII operons were usually complete and the afa genes were conserved, apart from minor sequence variation within afaEVIII (Supplementary Fig. 10). Long-read sequencing of CC23 isolate US26 resolved afaVIII on an 82-kb genomic island that was integrated at the tRNA-argW site. This hybrid pathogenicity-resistance island carried one additional VAG, agn43, which encodes antigen 43 (Ag43), a protein involved in biofilm formation, adhesion, and autoaggregation ${ }^{34}$, plus 10 genes predicted to confer resistance to beta-lactams, sulfonamides, phenicols, aminoglycosides, or mercury (Supplementary Fig. 11).

UPEC isolates harbor a diverse VAG repertoire. The UPEC isolates each contained between 164 and 382 VAGs, some of 


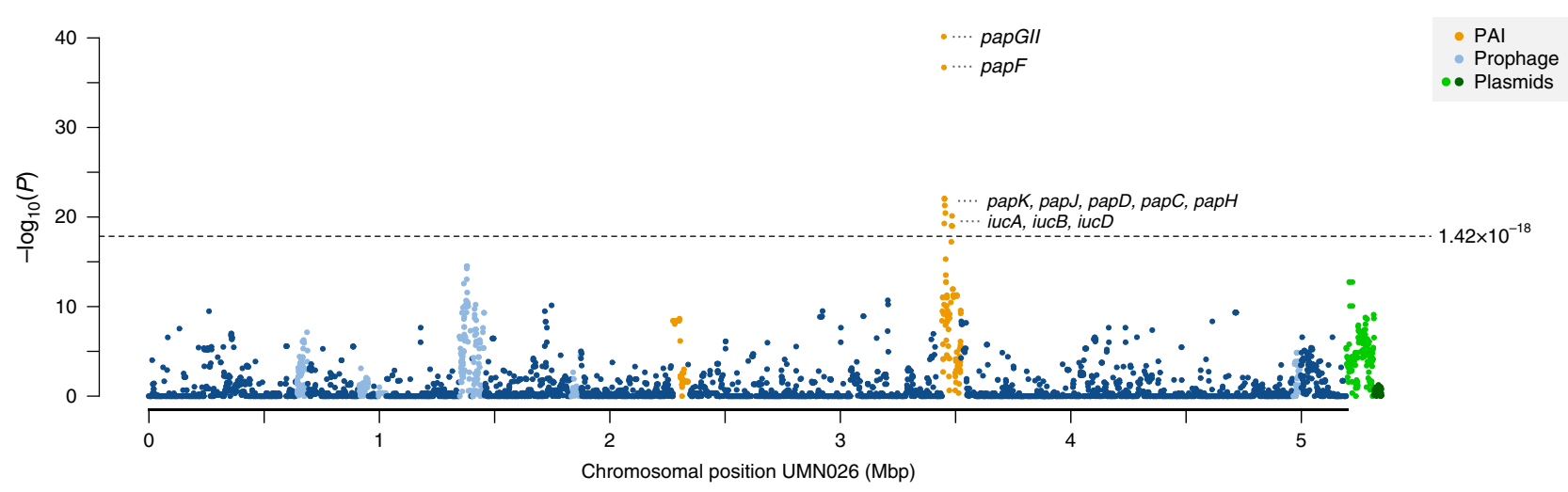

Fig. 2 Manhattan plot for pan-genome-wide associations for invasive vs. non-invasive UPEC. Data are based on 30,705 clusters of orthologous genes (COGs) identified in 722 UPEC isolates. Fecal isolates were not considered in this analysis because they present no urinary phenotype. The plot shows genes assigned to 4764 unique COGs identified in the genome of reference strain UMNO26 (CC69). Each dot represents one COG. The vertical axis denotes raw $P$ values of Fisher's exact statistics. To account for the effects of sample size and population structure, the genome-wide significance threshold (dotted line, $P=1.42 \times 10^{-18}$ ) was inferred from a simulated dataset using treeWAS. The horizontal axis gives the nucleotide position in the chromosome. COGs part of pathogenicity islands (PAIs), prophage regions, or the two plasmids of UMN026 are color-labeled, including the High Pathogenicity Island (HPI) and PAlUMNO26-phev, a papGIl-containing PAI. The remaining 25,941 COGs of the pan-genome that did not map to UMN026 were not pan-genomewide significant (Supplementary Fig. 21).

which were located on papGII+ PAIs (Fig. 4a, Supplementary Fig. 8a). VAGs identified on one or more of the 42 resolved papGII+ PAIs included iron uptake systems (iuc, ireA, iha, fbp, fec), fimbrial genes ( $f i m, u c l)$, invasins (tia, hek, hra2), toxins (cnfl, hly), autotransporters (sat, agn43), toxin-antitoxin modules (cdiA/cdiB), and sisA and $s i s B$, which in mice downregulate the kidney's immune response during E. coli infection ${ }^{35}$.

The number of VAGs correlated with the isolates' associated disease severity. Genomes from invasive UPEC isolates contained significantly more VAGs than did those from cystitis isolates, and genomes from cystitis isolates contained significantly more VAGs than did those from $A B U$ isolates (Fig. 4a). To account for this observation, cystitis isolates were analyzed separately from $A B U$ isolates. To examine functional correlations, VAGs were grouped by presumptive functional class (Supplementary Data 8 ). With VAGs stratified by functional class, as compared with either the cystitis or ABU isolates the invasive UPEC isolates had a higher prevalence of VAGs related to iron uptake and immune evasion/ modulation. In addition, as compared with $\mathrm{ABU}$ isolates, they had a higher prevalence of VAGs related to secretion systems/ autotransporters, adhesion/invasion (attributable to pap genes only), toxins, and bacteriocins (Supplementary Table 6a).

The number of VAGs varied significantly by phylogroup, analogous to the observed phylogroup-specific segregation of clinical phenotypes. Specifically, the number of VAGs was significantly higher in UPEC-associated phylogroups (B2, C, D, F) than in those associated with fecal isolates (A and B1) (Supplementary Fig. 12, Supplementary Table 7). Within phylogroup B2, the total number of VAGs did not differ significantly between invasive UPEC isolates and cystitis isolates, but was significantly higher among invasive UPEC isolates as compared to $\mathrm{ABU}$ isolates, a difference that is possibly attributable to the acquisition or loss of single PAIs (Supplementary Fig. 13, Supplementary Table 6b). By contrast, among papGII+ isolates the number of VAGs did not differ significantly by clinical source, i.e., between invasive UPEC, cystitis, ABU, or fecal isolates (Supplementary Table 8).

Iron acquisition systems are regarded as critical virulence factors of $\mathrm{UPEC}^{36}$. Of the 22 presumably partially redundant iron uptake systems described for E. coli $i^{37-39}$, the average number of such systems per isolate was significantly higher among invasive UPEC isolates (15.6) than among cystitis isolates (14.3), $\mathrm{ABU}$ isolates
(14.0), or fecal isolates (12.9) (Supplementary Table 9). papGII+ isolates carried, on average, 16.3 iron uptake systems (vs. 13.5 among papGII-negative isolates, $P<0.001)$. As noted generally for VAGs, the average number of iron uptake systems per isolate correlated with phylogenetic background, with the highest values for (invasiveness-associated) phylogroups B2 and F (Fig. 4b, Supplementary Table 9). Apart from iron acquisition systems, previous studies found associations with uropathogenicity for multiple other VAGs ${ }^{40-43}$. In agreement with these findings, many of these uropathogenicity-associated VAGs were here found in both invasive and non-invasive UPEC; and were associated with specific CCs rather than invasiveness (Supplementary Fig. 14).

Apart from papGII, only the iuc (i.e., aerobactin) locus, which in $E$. coli consists of six genes (shiF, iucA, iucB, iucC, iucD, iutA), reached pan-genome-wide statistical significance for invasive vs. non-invasive UPEC isolates $\left(P=7.6 \times 10^{-21}\right)$. The iuc locus occurred in $73 \%$ of invasive UPEC isolates, vs. $36 \%$ of cystitis isolates $(P<0.001, \mathrm{OR}=4.7)$ and $48 \%$ of $\mathrm{ABU}$ isolates $(P<0.001$, $\mathrm{OR}=2.9$ ). It also was associated specifically with papGII, occurring in $86 \%$ of papGII+ isolates vs. $33 \%$ of pap GII-negative isolates $(P<0.001, \mathrm{OR}=13.0)$. A complete iuc locus $(100 \%$ sequence coverage) was identified in $92 \%$ of the $482 i u c+$ isolates. In the remaining $38 i u c+$ isolates the $i u c$ locus either could not be resolved (15 isolates) or showed disruptions (23 isolates). We identified three distinct, highly conserved iuc locus architectures. These included (i) shiF/iuc/iutA1, which was usually associated with type II papGII+ PAIs; (ii) shiFp/iuc/iutA2, which was part of large IncFII plasmids and corresponds to the iuc 5 locus described in Klebsiella isolates ${ }^{44}$; and (iii) shiF/iuc/iutA2, which was part of the widely distributed $\mathrm{PAI}_{\mathrm{ABU} \text { 83972-phev-like islands or, less }}$

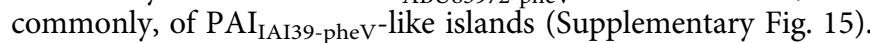
The distribution of the non-aerobactin iron uptake systems by clinical phenotype and phylogroup is described in Supplementary Note 3 and Supplementary Table 10.

papGII+ sublineages within $\mathrm{CC} 69, \mathrm{CC} 95$, and $\mathrm{CC} 73$. The three pandemic UPEC lineages CC69, CC95, and CC73 comprised, collectively, $42 \%(301 / 722)$ of the UPEC isolates and $68 \%$ (229/ 333 ) of the papGII+ isolates, as compared with $23 \%$ of the fecal isolates. Each of these lineages exhibited gain, loss, and/or rearrangements of papGII+ PAIs, which segregated by lineage. 


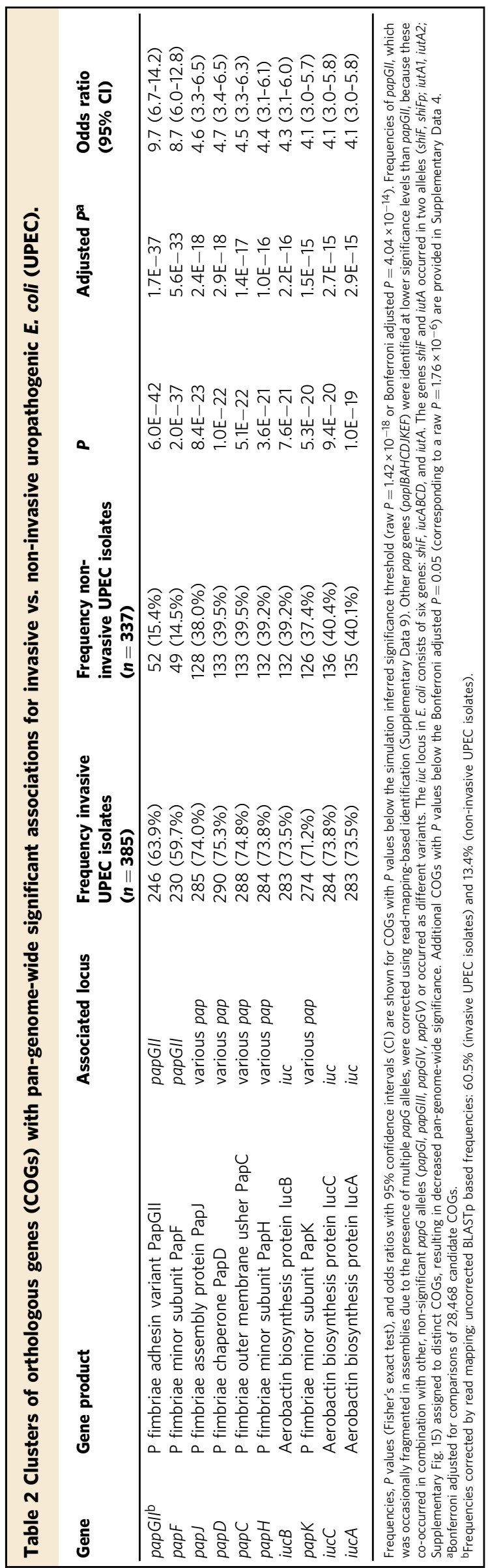

Within CC69, 53 of 54 papGII+ isolates formed a monophyletic clade and shared a conserved PAI. This suggests that this clade emerged after a single acquisition event involving a type II papGII+ PAI in a common cladal ancestor (Fig. 5).

Similarly, within CC95, isolates of the papGII+ sublineage shared a conserved type V PAI. Occurrence of the papGII+ PAI at either the tRNA-pheU or tRNA-pheV site within one branch suggests possible excision and re-integration events within CC95 isolates (Supplementary Fig. 16). The population structure, supported by high-confidence bootstrap values, suggests that the PAI was present in the most recent common ancestor of all CC95 isolates but subsequently was lost in one branch (Fig. 5). Interestingly, this papGII-negative branch, which corresponds with so-called CC95 subgroup $\mathrm{B}^{45}$, consisted mainly of noninvasive UPEC isolates and contained a sublineage characterized by integration of a papGIII-containing PAI at the tRNA-leuX site.

Within CC73, all four papGII+ sublineages (here termed CC73-L1, -L2, -L3, and -L4) were part of a single large monophyletic branch characterized by the presence of iuc (Fig. 5). The occurrence of different papGII+ PAI types within this iuccontaining branch suggests different papGII+ PAI acquisition events within each sublineage. Alternatively, the different sublineages might have evolved from a common ancestor that contained multiple papGII+ PAIs, such as seen in reference pyelonephritis isolate CFT073, which is phylogenetically similar to isolates of this branch and harbors distinct papGII+ PAIs at its two tRNA-phe sites.

The four papGII+ CC73 sublineages differed for the composition and insertion site(s) of their PAIs. Specifically, several isolates within sublineages CC73-L1 and CC73-L2, carried a type V PAI at the tRNA-pheU site, whereas others carried type III PAIs, including

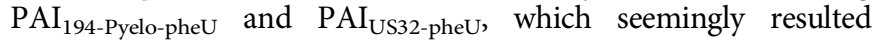
from recombination between a type $\mathrm{V}$ PAI and a papGIIIcontaining PAI (Supplementary Fig. 17). By contrast, isolates within sublineage CC73-L3 carried a type II PAI at the tRNA-pheV site, and those within sublineage CC73-L4 carried a type IV PAI. This PAI was integrated adjacent to a $\mathrm{PAI}_{\mathrm{ABU}}$ 83972-pheV-like island at the tRNA-pheV site.

Notably, isolates from the large iuc-containing branch within CC73 carried one of two iuc locus configurations, i.e., either (1) shiF/iuc/iutA1, on type II papGII+ PAIs (found in CC73-L3), or (2) shiF/iuc/iutA2, on $\mathrm{PAI}_{\mathrm{ABU}}$ 83972- $\mathrm{phev}^{-l i k e}$ islands (found in CC73-L1, CC73-L2, and CC73-L4). The latter was also found in isolates from sublineages associated with papGII-negative, noninvasive UPEC isolates, such as prototypic ABU strain ABU83972 and probiotic strain Nissle1917. These two iuc-containing PAIs might have been acquired independently or could have evolved from the same PAI, after its acquisition by a common CC73 ancestor.

papGII+ sublineages within the pandemic UPEC lineage CC131. The recently emerged lineage CC131 (dominated by ST131) is currently the leading cause of multi-drug-resistant E. coli UTI and bloodstream infections ${ }^{12,46}$. Although our main dataset comprises isolates collected over almost 40 years, it includes few CC131 isolates $(n=59)$, precluding a robust analysis of this lineage. Accordingly, to investigate the distribution of papGII within CC131 we combined these 59 CC131 isolates with 1017 additional publicly available CC131 isolates, which had, however, limited associated metadata (Supplementary Table 11, Supplementary Data 10). This analysis identified in all three main CC131 clades (A, B, and C) sublineages enriched with papGII+ isolates (Supplementary Fig. 18). The largest papGII+ fractions occurred within both subclade C2 (i.e., H30Rx; 216/521 isolates, 41\%) and the fimH27-subclade within clade B (17/22 isolates, 77\%). 

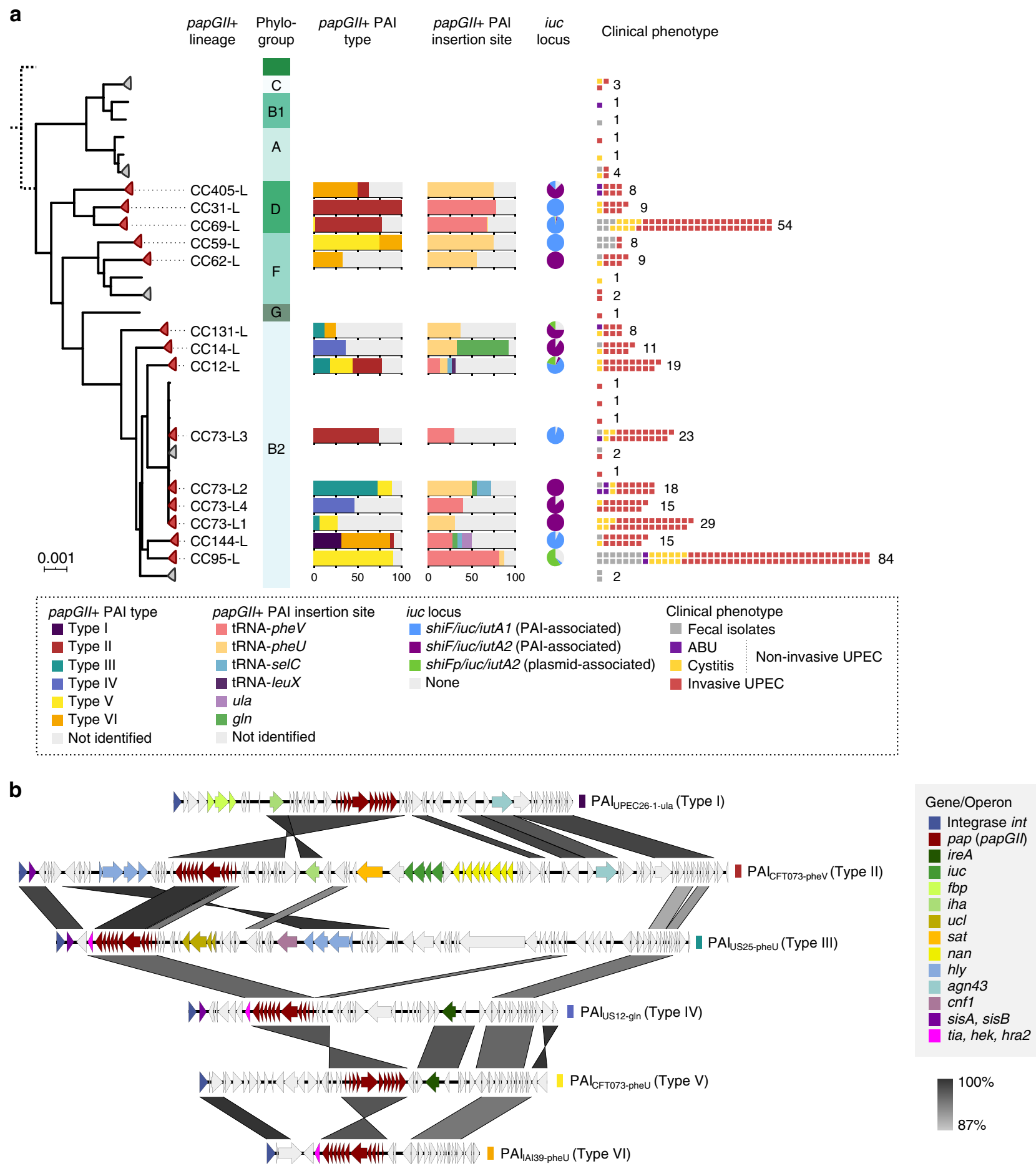

Fig. 3 Genetic characterization of papGII+ E. coli lineages and papGII+PAls. a Maximum-likelihood phylogenetic tree of 333 papGII+ isolates based on 192,889 variable sites identified in a core genome alignment (2.573 Mbp). Phylogenetic lineages, defined by patristic distances, are collapsed on single nodes (indicated with triangles). Fourteen papGll+ lineages with $>5$ isolates (red triangles) were identified and named after their clonal complex $(+\mathrm{L})$. Isolates in CC73 were investigated on an additional level of hierarchy and assigned to four papGII+ lineages (CC73-L1 to -L4) to account for the subclonal population structure with distinct characteristics. Each papGIl+ lineage is labeled with the proportion of papGll+ pathogenicity island (PAI) types and insertion sites when their identification was possible. The presence of papGII+ PAI types was identified in complete or near-complete assemblies or predicted using a read-mappingbased approach. Fragmented assemblies, lack of resolved reference PAls, or sequence deletions/insertions sometimes prevented the determination of the specific papGII+ PAI family type and insertion site (shown in gray). The proportion of isolates carrying PAI- or plasmid-associated iuc loci, the frequency of clinical phenotypes, and the total number of isolates are shown. The branch length of the outgroup (papGII-negative isolate 495_PUTI_Fec, clade I) was reduced (dashed line). The scale bar indicates the number of substitutions per site in the core genome alignment. A tree with expanded nodes and bootstrap values is shown in Supplementary Fig. 16. b Genetic organization of representative PAls of the six identified papGII+ PAI types. The papGIl operon, integrase gene, and virulence-associated genes are highlighted. The gradient scale shows the level of nucleotide identity. PAI sequences were compared and visualized using EasyFig110. The genetic organization of all 42 resolved papGII+ PAls is shown in Supplementary Fig. 22. 
a

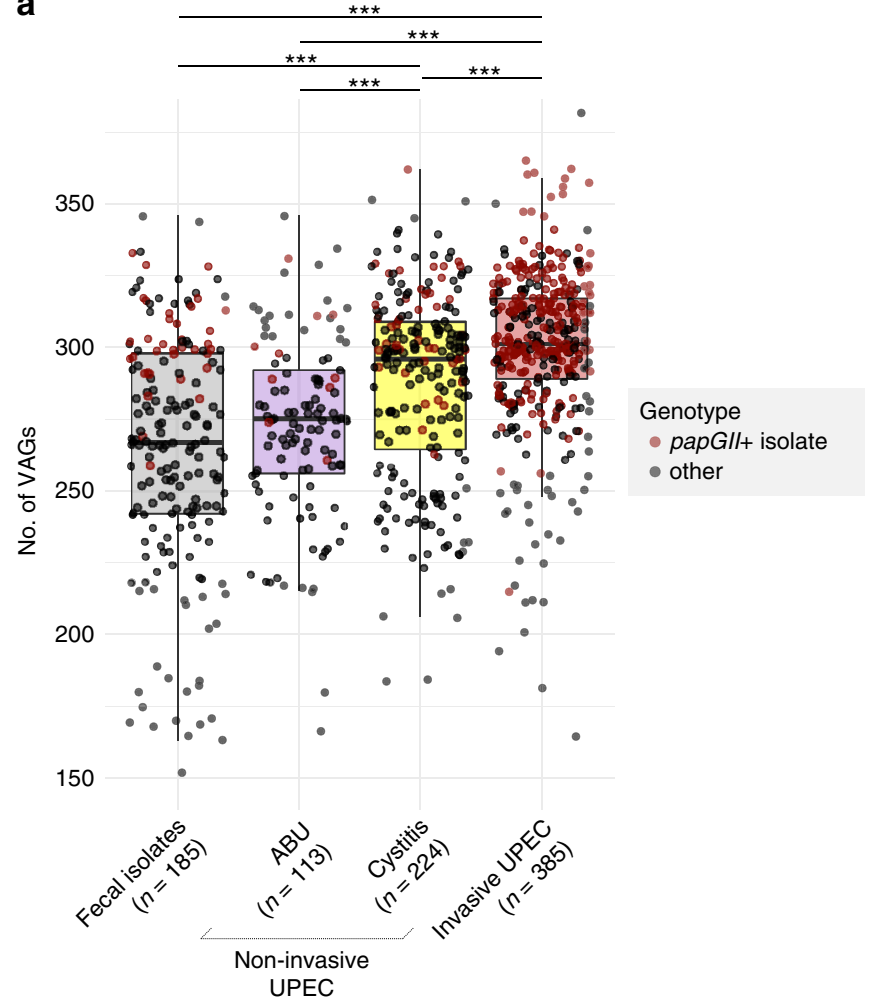

b
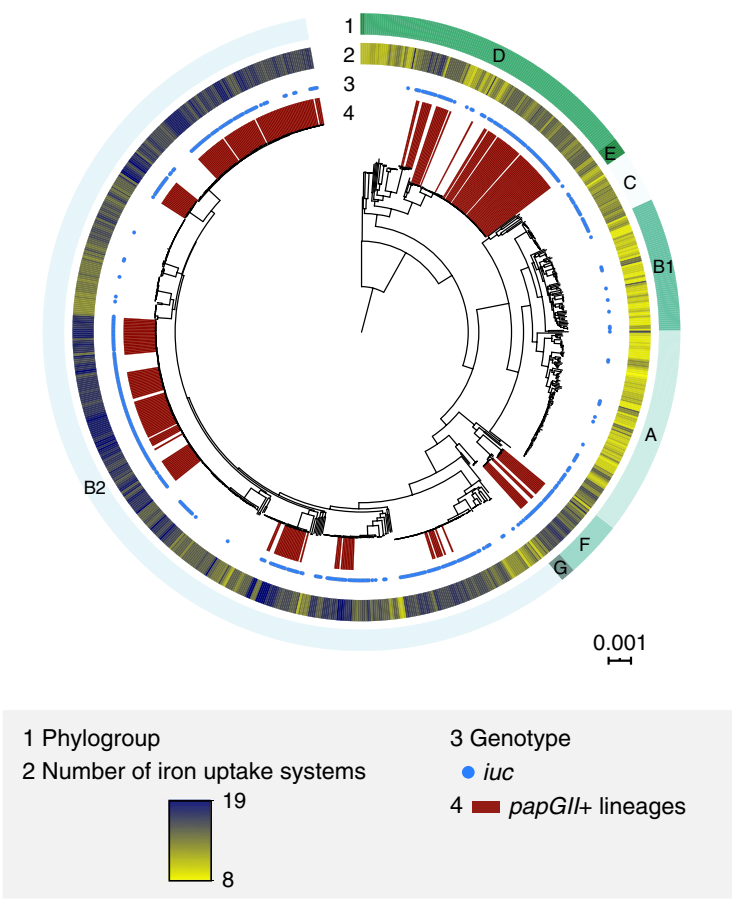

Fig. 4 Number of virulence-associated genes (VAGs) and distribution of iron uptake systems. a Boxplots showing the number of VAGs per isolate by clinical phenotype (fecal isolates, non-invasive UPEC isolates (asymptomatic bacteriuria (ABU), cystitis), and invasive UPEC isolates). papGII+ isolates are indicated as red dots. Asterisks indicate significant differences ( ${ }^{\star \star \star} P<0.001$, two-sided Mann-Whitney $U$ test, Bonferroni-corrected). Exact $P$ values are reported in Supplementary Fig. 13. Boxplot center lines: median; box limits: upper and lower quartiles; whiskers extend from the hinges to the highest and lowest values that are within 1.5 $\times \mathrm{IQR}$ of the hinges. Source data are provided in Supplementary Data 8. b Number of iron uptake systems (ring 2) per isolate and presence of iuc (ring 3) visualized on the phylogenetic tree. Twenty-two different systems involved in iron uptake were identified in our dataset, with 8-19 systems found per isolate. Phylogroups (ring 1) and isolates part of papGII+ lineages (ring 4) are labeled. The tree was visualized using iTOL ${ }^{89}$.

Despite its recent emergence, CC131 appeared to have undergone multiple acquisitions of papGII+ PAIs. Among the 21 papGII+ CC131 isolates with complete or near-complete assemblies, 20 contained a type III and one a type IV papGII+ PAI (Supplementary Table 12, Supplementary Fig. 19). Among the remaining papGII+CC131 isolates with available read data, a read-mapping-based analysis identified type III papGII+ PAIs in multiple isolates of clades A, B, and C, and, sporadically, type II, type IV, type V, and type VI papGII+ PAIs in isolates of clades B and C (Supplementary Fig. 18, Supplementary Data 12). Among the 192 CC131 UPEC isolates with available clinical phenotype data, papGII was significantly more prevalent among invasive UPEC isolates $(67 / 133,50 \%)$ as compared to non-invasive UPEC isolates $(7 / 59,12 \%$ : $\mathrm{OR}=7.5, P<0.001)$.

\section{Discussion}

In this study, we analyzed 907 E. coli isolates from individuals with $\mathrm{ABU}$, cystitis, pyelonephritis, or urinary-source bacteremia, as well as from feces, thereby deriving a high-resolution population structure of UPEC associated with these different clinical contexts. The observed phylogenetic clustering of invasive UPEC isolates underlines the importance of the corresponding lineageassociated genetic determinants in UTI pathogenesis. Our large collection size provided sufficient statistical power to establish a genome-wide association of papGII with invasive disease, suggesting a causal relationship. Associations of most other genes were rejected by stringent statistical corrections for multiple testing and population structure.
The gene papGII has been associated epidemiologically with pyelonephritis and urinary-source bacteremia in directed, usually PCR-based studies ${ }^{19-22,47}$, and was shown experimentally, with varying degrees of rigor, to contribute to kidney infection in murine and monkey models ${ }^{48-50}$. A key role for papGII in the pathogenesis of human pyelonephritis was confirmed recently by the finding that knock-in of papGII was sufficient to enable the iuc-positive but normally non-pathogenic $E$. coli strain ABU83972 (CC73, phylogroup B2) to cause pyelonephritis in humans ${ }^{23}$. In that study, PapGII was shown to enter kidney cells and to trigger renal tissue inflammation by reprogramming host gene expression ${ }^{23}$. These findings, together with ours, support a site (i.e., kidney)-specific and, hence, pathotype-defining role for papGII. However, our data also identify a comparatively rare locus, specifically afaVIII, as potential marker of invasive UPEC.

Apart from papGII, only the iuc locus exhibited a significant pan-genome-wide association with invasive vs. non-invasive UPEC. This suggests that iron scavenging, as mediated specifically by the aerobactin system (among multiple E. coli siderophores), is particularly important for tissue and bloodstream invasion during UTI. Abundant evidence supports both a critical role of iron uptake for UPEC and partial functional redundancy among the multiple $E$. coli iron acquisition systems ${ }^{36}$. Here, iron uptake was the virulence factor class most significantly enriched in invasive UPEC isolates, as compared to non-invasive UPEC or fecal isolates.

Previous studies identified $f y u A$ (yersiniabactin siderophore) and chuA (heme-binding protein) as important pathogenicity determinants of ExPEC and UPEC ${ }^{16,42}$. Although here, after 
adjustment for multiple comparisons and population structure, fyuA and chuA did not discriminate between invasive and noninvasive UPEC isolates, this does not exclude a role in general uropathogenicity for them. For example, we showed a clear association of $f y u A$ and $c h u A$ with phylogroups B2, C, D, and F, which together harbored $94 \%$ of invasive UPEC isolates and $75 \%$ of non-invasive UPEC isolates. By contrast, chuA was absent and fyuA was uncommon (prevalence 20-35\%) in commensalassociated phylogroups $\mathrm{A}$ and $\mathrm{B} 1$, suggesting that $f y u A$ and chuA might play a role in urinary-tract colonization for both invasive and non-invasive UPEC. Future studies with specific datasets from fecal and urinary $E$. coli isolates could resolve the bacterial determinants of commensal vs. invasive and noninvasive UPEC.

Although we focused on the role of bacterial determinants in UTI pathogenesis, predisposing host factors also are critically important. Here, some of the putative invasive UPEC isolates that lacked recognized VAGs (including papGII) may have been misclassified based solely on clinical criteria, and actually caused their invasive infection due to host defense defects rather than heightened pathogenic potential. Debilitated hosts are often infected by $E$. coli strains with low intrinsic virulence, such as those from phylogroup A or B1 and that produce fewer virulence factors $^{51}$, and $p a p G$ is less common among invasive isolates from compromised as compared with non-compromised patients ${ }^{52}$.

Conversely, a substantial fraction of the present cystitis and $\mathrm{ABU}$ isolates, including strains that asymptomatically colonized the urinary tract over months ${ }^{53}$, carried papGII, without causing invasive infection. Apart from possible stochastic effects, this may reflect in part the known variation in host susceptibility to UTI in relation to specific host genetic polymorphisms ${ }^{54}$; conceivably, these isolates' corresponding source hosts may have been more resistant to developing invasive UTI. In addition, the study's clinical phenotype assignments may have misrepresented some strains' virulence potential. For example, patients classified as having cystitis may have received prompt antibiotic treatment that prevented progression of papGII+ isolates to the kidneys, or their urine samples might have been collected before symptoms of pyelonephritis developed.

The observed preferential occurrence of papGII+ PAIs in expanded lineages associated with invasive UPEC is similar to observations of acquisitions of PAIs or plasmids linked to the clonal expansion of atypical EPEC ${ }^{9}$ and ETEC lineages ${ }^{10}$. Whereas most ETEC and atypical EPEC lineages in those studies were from phylogroups $\mathrm{A}$ and $\mathrm{B} 1$, here all papGII+ lineages belonged to phylogroups B2, D, and F. Consistent with previous findings ${ }^{4}$, our data demonstrate that isolates of phylogroups B2, $\mathrm{C}, \mathrm{D}$, and F carry greater numbers of VAGs than do isolates of other phylogroups, even in the absence of papGII+ PAIs, suggesting that such VAGs provide the genetic basis required to colonize extraintestinal sites such as the human urinary tract. This ability to colonize extraintestinal sites might be a precondition for the stable maintenance of acquired papGII+ PAIs and clonal expansion.

The spread of AMR among pathogenic E. coli poses a significant threat to public health. In particular, the emergence of papGII+ lineages within ST131 clades $\mathrm{C} 1$ and $\mathrm{C} 2$, which were shown to have high levels of AMR against commonly used antimicrobials to treat invasive UTI (ciprofloxacin, 3rd-generation cephalosporines, trimethoprim-sulfamethoxazole $)^{55,56}$, is concerning. In addition to bacterial virulence and host factors, AMR and associated treatment failure may impact the outcome of UTI, if not the patient's initial presentation. Here, invasivenessassociated sublineages were found among both multi-drug resistant clones (CC69 and CC131) and clones that typically exhibit broad antibiotic susceptibility (CC73, CC95 $)^{12,13}$. Regardless of their usual AMR status, all such invasiveness sublineages shared the presence of papGII or afaVIII.

Considering the widespread presence of papGII+ PAIs and their stable maintenance in successful $E$. coli lineages, we speculate that PapGII confers a niche-specific selective advantage. P fimbriae with PapGII might contribute to urinary-tract colonization and thereby to pathogen transmission through shedding. Alternatively, PapGII might contribute to gut colonization; indeed, phylogroup B2 isolates that produce $\mathrm{P}$ fimbriae have been associated with increased persistence in the human intestine $\mathrm{e}^{57,58}$. PapGII might also play a role in the colonization of zoonotic niches. Interestingly, papGII was found in up to $60 \%$ of avian pathogenic E. coli isolates from live or diseased poultry ${ }^{59-61}$. Human intestinal acquisition of UPEC/ExPEC has been associated with animal contact, consumption of high-risk food (including seafood, raw meat, or vegetables), and direct transmission between humans ${ }^{13,62}$.

Previous studies demonstrated that UTI occurrence is linked to the relative abundance of the causal clone in the host's gut microbiota, and modulation of the gut microbiota has been suggested as a potential strategy to prevent $\mathrm{UTI}^{63,64}$. Here, papGII + isolates from feces genetically resembled clinical papGII+ isolates, supporting the concept that the human gut is a reservoir of papGII+ isolates and that carriers might be predisposed to invasive UTI. In our dataset, papGII+ isolates (i.e., putative uropathogens) constituted $\sim 20 \%$ of all fecal E. coli isolates.

Our study has notable limitations and strengths. Limitations include (i) an intrinsic sampling bias due to the variation in the distribution of $E$. coli clones, both over time and among different human populations; (ii) a potential underestimation of the contribution of rare variants or the combined effects of variants due to our stringent statistical adjustment by GWAS, leading to failure to detect true associations; (iii) inaccessibility of data on predisposing host factors, and (iv) reliance on observation/correlation, not experimentation. In addition, gene presence does not necessarily imply functional expression, and in vivo gene expression levels are unknown for the present study isolates. Notably, regulation of pap expression is complex ${ }^{65,66}$. Strengths include (i) the large number of study isolates with welldocumented clinical phenotypes, (ii) the unbiased approach to identify genome-wide associations of genes and lineages with specific clinical syndromes, and (iii) use of a robust phylogeny in combination with long-read sequencing data to elucidate key evolutionary events.

In summary, our findings demonstrate that different UPEC lineages tend to behave as either invasive or non-invasive pathogens. Whereas a combination of multiple VAGs is likely required to colonize the urinary tract, papGII is specifically associated with invasiveness. A few papGII+ lineages account for most invasive UTIs across decades and continents, with recent or ongoing papGII+ PAI acquisition events likely leading to the emergence of new papGII+ lineages. These findings have implications for our understanding of the pathogenesis of invasive UPEC and, hence, for potential surveillance and control measures.

\section{Methods}

Bacterial isolates and genomes of the main dataset. Genomes of 907 E. coli isolates from multiple collections were analyzed. These included 185 fecal isolates from human samples and 722 urinary-tract source extraintestinal isolates from human subjects with $\mathrm{ABU}(n=113)$, cystitis $(n=224)$, pyelonephritis $(n=73)$, or urinary-source bacteremia $(n=312)$. Of these genomes, 738 originated from 13 publicly available collections (Supplementary Table 1). These were supplemented with genomes of 18 reference strains and 151 isolates that were sequenced as part of this study, including isolates from the collections LtABU, UZA_uro, MVAST_ABU, and MC_pye. Strain details are listed in Supplementary Data 1.

LtABU collection. The LtABU collection comprises isolates associated with long-term ABU obtained between 2017 and 2018 from non-catheterized residents 
CC69
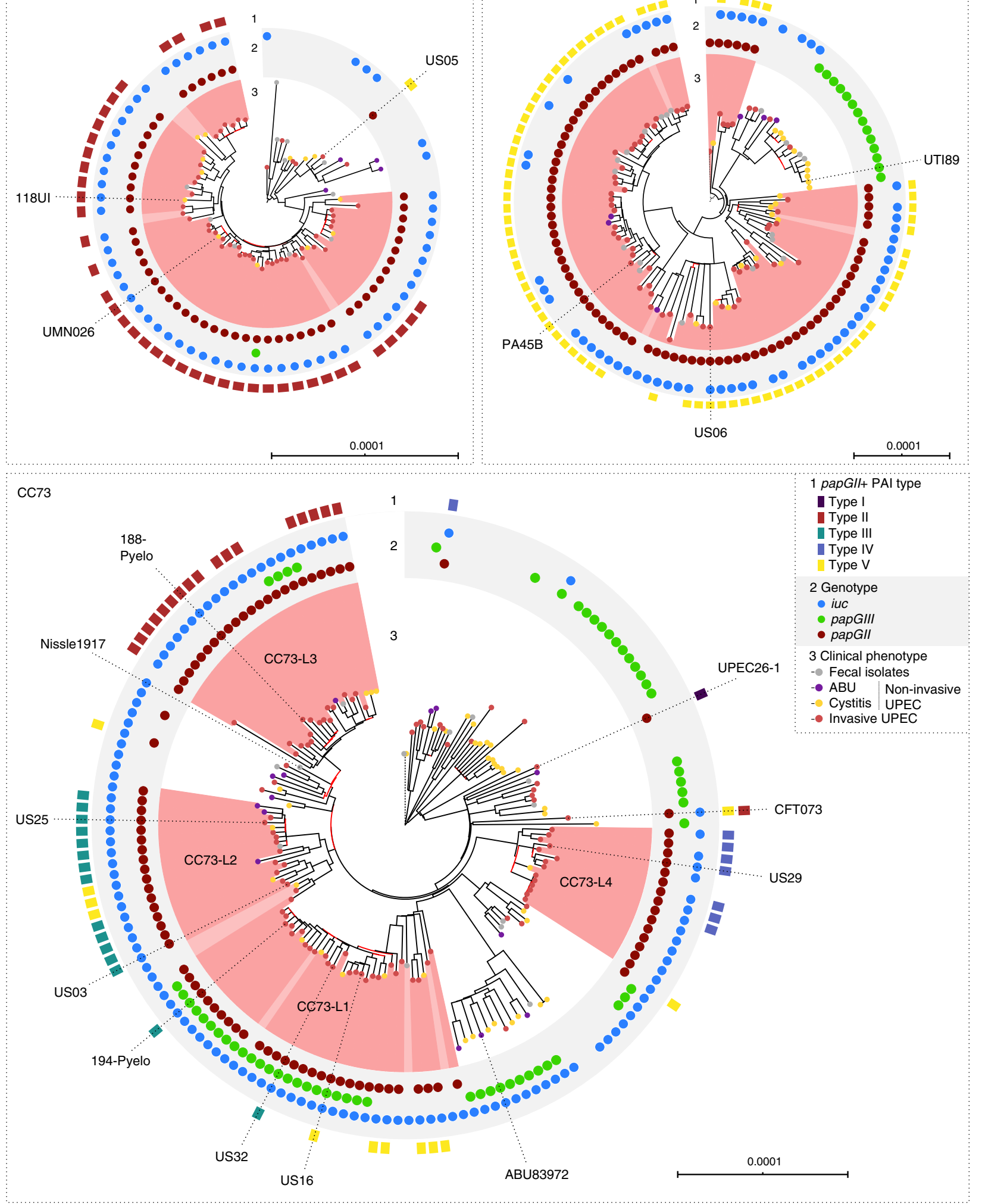

( $>65$ years of age) of two long-term care facilities (OCMW Destelbergen and WZC Immaculata Edegem) in Belgium. A total of 237 participants were screened for bacteriuria $\left(>10^{5} \mathrm{CFU} / \mathrm{ml}\right)$ at up to four consecutive sampling timepoints over three months: at baseline and after $\sim 2,10$, and 12 weeks. Bacterial species were identified using MALDI-TOF. The clonal relationship of consecutive E. coli bacteriuria isolates from the same patients was analyzed using random amplification of polymorphic DNA (RAPD) profiling. The RAPD assay consisted of two PCR reactions containing primer 1247 (5'-AAGAGCCCGT-3') or 1283
CC95

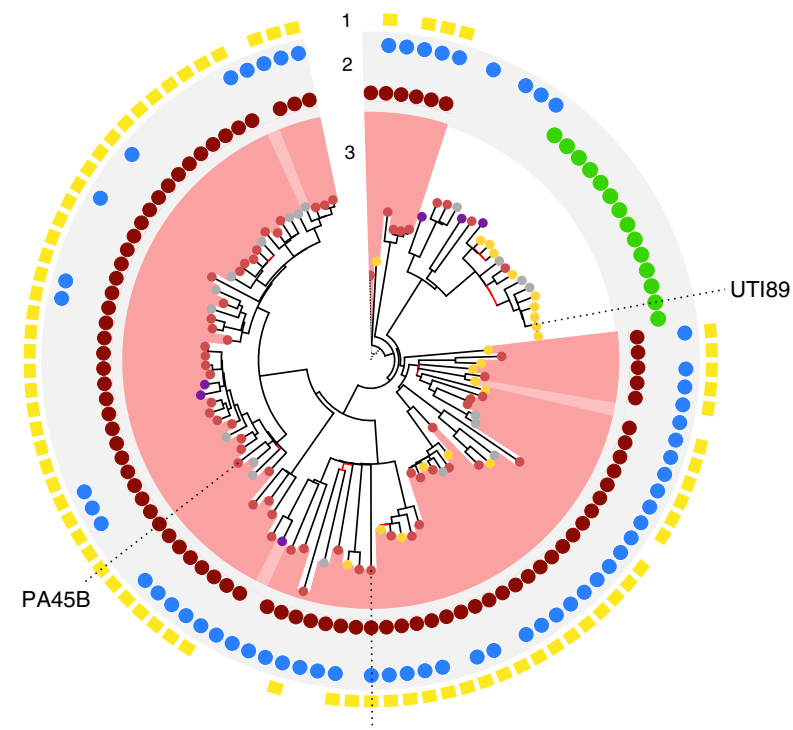

US06

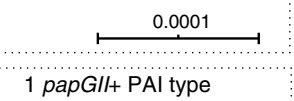

Type V

2 Genotype

- iuc

Non-invasive

Cystitis UPEC

Invasive UPEC
(5'-GCGATCCCCA-3'), respectively, as described by Nielsen et al. ${ }^{67}$. Amplification was performed in $27 \mu \mathrm{L}$ reactions with $1.5 \mu \mathrm{M}$ primer, $0.2 \mathrm{mM}$ dNTPs, $1.5 \mathrm{U}$ SuperTaq polymerase (SphaeroQ), the supplied polymerase buffer, and $2 \mu \mathrm{l} 100$ fold diluted overnight culture. Cycling conditions were $15 \mathrm{~min}$ at $95^{\circ} \mathrm{C}, 35$ cycles of $1 \mathrm{~min}$ at $94^{\circ} \mathrm{C}, 1 \mathrm{~min}$ at $38^{\circ} \mathrm{C}$ (reaction primer 1247 ) or $36^{\circ} \mathrm{C}$ (reaction primer 1283), and $2 \mathrm{~min}$ at $72^{\circ} \mathrm{C}$, followed by a final extension for $10 \mathrm{~min}$ at $72^{\circ} \mathrm{C}$. Amplification products were separated on $1.5 \%$ agarose gels in TBE buffer $(1 \mathrm{~h}$, $150 \mathrm{~V}$ ), stained with GelRed (Biotium), and visualized under UV light (BioRad 
Fig. 5 Phylogenetic trees of isolates belonging to pandemic UPEC lineages CC69, CC95, and CC73. Midpoint rooted maximum-likelihood phylogenies based on core genome alignments (CC69: 76 isolates, 25,753 variable sites in 4.006 Mbp core genome; CC95: 107 isolates, 17,674 variable sites in 4.054 Mbp core genome; CC73: 164 isolates, 25,939 variable sites in $3.857 \mathrm{Mbp}$ core genome). Clinical phenotypes are labeled at the branch tips (ring 3). The presence of papGII, papGIII, and iuc is shown (ring 2). When identification was possible, papGII+ PAI types are labeled (ring 1). Fragmented assemblies, lack of resolved reference PAls, and sequence deletions or insertions within PAls sometimes prevented the determination of the specific papGII+ PAI type. Isolates part of papGII+ lineages are shaded in red; isolates part of the same lineages but lacking papGII in bright red. Isolates with complete or nearcomplete genomes used to investigate the genetic context of papGIl are annotated. Red branch lines indicate nodes with bootstrap values <70. Branch lengths of distantly related isolates (outgroup) are reduced and indicated as dashed lines. The papGII-negative subclade in CC95 corresponds to the previously defined subgroup B (serotype O18:H7) ${ }^{45}$. The trees were visualized using $\mathrm{iTOL}^{89}$.

ChemiDoc). Long-term $\mathrm{ABU}$ was defined here as at least three consecutive positive urine cultures covering a period of at least 10 weeks from participants without signs or symptoms of a urinary-tract infection and resulting in the isolation of clones with identical RAPD fingerprints. Forty-three long-term E. coli ABU isolates from 42 participants (median age 86, range 70-97) were identified and included in this study. One subject carried two E. coli clones (LtABU20, LtABU24) with distinct morphologies at three consecutive sampling points reaching maximum concentrations of $>10^{7} \mathrm{CFU} / \mathrm{ml}$ each. Most of the isolates originated from female participants (41/42) with incontinence (39/41). A comprehensive analysis of bacteriology and risk factors associated with $\mathrm{ABU}$ at one of the two collections sites was published previously ${ }^{53}$.

UZA_uro collection. The UZA_uro collection comprises urosepsis isolates obtained from the Antwerp University Hospital UZA. Hospital records from 2015 to 2018 were screened for non-compromised patients with community-acquired sepsis who had positive urine and blood cultures with matching $E$. coli isolates (i.e., identical antibiotic susceptibility patterns) separated in time by $\leq 1$ week. Immunocompromising conditions, surgery, pregnancy, urological intervention, and putative hospital-acquired infection were exclusion criteria. Sepsis symptoms were defined based on the SIRS criteria ${ }^{68}$. Infection was considered community-acquired when the sampling date of either blood or urine culture was no more than 2 days after admission to the hospital. Non-duplicate urosepsis isolates from 30 such patients (median 75.5 years, range $0-92$ ) were available.

MVAST_ABU collection. The MVAST_ABU collection comprises asymptomatic bacteriuria isolates from male patients at the Minneapolis Veterans Affairs Medical Center (MVAMC) without long-term care facility residence in the previous year. The collection is a subset of isolates from various clinical sources described by Drekonja et al. ${ }^{69}$.

MC_pye collection. The MC_pye collection comprises urine isolates from female patients ( $>18$ years of age) with acute, uncomplicated pyelonephritis originally collected between 1994 and 1996 during a multi-center study in the United States ${ }^{70,71}$. Inclusion criteria were flank pain/tenderness, fever $\left(>38^{\circ} \mathrm{C}\right)$, and pyuria. Exclusion criteria included immunocompromised status, hospital admission, urologic abnormalities, and diabetes. Of the 70 isolates of the MC_pye collection, 39 were sequenced here and 31 had publicly available genomic data.

Publicly available collections. Publicly available genomic data for 875 isolates from 13 collections were considered for inclusion. Thirty-eight isolates were excluded due to low sequence quality $(n=25)$, inaccessible or corrupted read files $(n=8)$, inconclusive metadata $(n=4)$, or doubtful species $(n=1)$. Sixty-four isolates from the KTE_and Rec_cys collections were excluded due to duplicate sequencing of reported same-clone isolates from the same patients. The KTE collection originally comprised 48 urinary isolates from 47 cystitis psatients, 81 fecal isolates from the same patients, which partially $(n=40)$ corresponded to the infecting clone, and 67 fecal isolates from patients who never had a UTI (control). The 40 fecal isolates that were reported to match to the cystitis clone in the same patient ${ }^{18}$ were excluded. The Rec_cys collection originally comprised 43 E. coli genomes obtained through consecutive sampling from 14 women with recurrent cystitis $^{43}$. Putative same-clone isolates were excluded. Thirty-five isolates from the PUTI_cys, MC_pye, and MN_fec collections were excluded to correct for sampling bias. PUTI_cys, MC_pye, and MN_fec comprised isolates originally selected for sequencing based on presumptive ExPEC status (i.e., presence of at least two of the genes $p a p A H$ and/or papC, sfalfocDE, afa/draBC, iutA, and $k p s M I I$ ), with an ExPEC:non-ExPEC target ratio of approximately one ${ }^{72}$. For our analysis, strains were randomly sub-selected to reflect the unbiased ExPEC/non-ExPEC ratio of the source collection. Descriptions of all collections and details on exclusions are provided in Table 1, Supplementary Table 1, and Supplementary Note 1.

Bacterial genomes of the CC131 dataset. Assemblies from CC131 isolates of the main dataset were analyzed in the context of publicly available CC131 wholegenome sequences including assemblies of 92 isolates from children with febrile UTI $^{73}$ defined here as invasive UPEC isolates, 10 urosepsis isolates ${ }^{17}, 16$ cystitis isolates $^{74}, 52$ isolates of various clinical phenotypes ${ }^{46}$, and 799 isolates without available metadata randomly downloaded from Enterobase ${ }^{75}$. Forty-eight complete or near-complete genomes of CC131 isolates identified on NCBI were also included in the analysis. Details of all isolates included in the CC131 dataset are provided in Supplementary Data 10 and collections are described in Supplementary Table 11.

Short-read sequencing. DNA was extracted from overnight liquid cultures (single colonies inoculated in $4 \mathrm{~mL}$ Mueller Hinton broth) using the MasterPure Purification Kit (Epicentre). Libraries were prepared using Nextera XT (Illumina), and sequencing performed on the Illumina MiSeq platform with $2 \times 250$ bp paired-end chemistries.

Long-read sequencing. Twenty-four isolates (Supplementary Data 5) were additionally sequenced using single-molecule real-time (SMRT) technology (Pacific Biosciences). DNA was extracted using the MagAttract HMW DNA Kit (Qiagen) and sheared to $\sim 8-10 \mathrm{~kb}$ using g-TUBE (Covaris). Libraries were prepared using the PacBio SMRTbell template preparation kit version 1.0 and pooled libraries of tagged isolates sequenced using the PacBio Sequel system.

De novo assemblies and annotation. Short-read data of 151 in-house sequenced isolates were pooled with raw reads downloaded from publicly available sources (NCBI's Sequence Read Archive or National Genomics Data Center, 724 isolates). Reads were trimmed using TrimGalore v0.4.4 (https://github.com/FelixKrueger/ TrimGalore) and assembled de novo with Spades v3.13.0 $0^{76}$. When long-read sequencing data were available, assemblies were obtained using HGAP $4^{77}$ with default settings with subsequent short-read polishing with Pilon implemented in Unicycler v0.4. $8^{78}$ and compared to hybrid assemblies performed with Unicycler v0.4.8 using default settings. The best assembly based on the number of contigs and N50 was used for further analyses. HGAP assemblies, which usually did not recover plasmid sequences, were combined with plasmid assemblies obtained from hybrid assemblies in Unicycler (see Supplementary Data 6). Contigs representing plasmids were predicted using MLPlasmids ${ }^{79}$ and replicons identified using the PlasmidFinder database ${ }^{80}$ in ABRicate v0.9.3 (https://github.com/tseemann/abricate) (minimum sequence coverage/identity 70/90\%). Pre-assembled data were downloaded from NCBI when raw read data were not accessible (17 reference strains and 15 isolates of collection Rec_cys, see Supplementary Data 1). Assembly quality was assessed using Quast $^{81}$. Genomes of all 907 isolates were annotated using Prokka v1.13.3 $3^{82}$

Phylogenetic analysis. To generate core genome alignments, collinear blocks (i.e. homologous genomic regions free from internal rearrangements) in assemblies were identified and aligned using Parsnp v1.283. Separate core genome alignments were generated for the main dataset (907 isolates, reference genome UMN026, GCA_000026325.2) and isolates belonging to CC69 (76 isolates, reference genome UMN026, GCA_000026325.2), CC73 (164 isolates, reference genome CFT073, GCA_000007445.1), CC95 (107 isolates, reference genome UTI89, GCA_000013265.1), and CC131 (1076 isolates, reference genome JJ1886, GCA_000493755.1). Prophage regions in reference genomes were identified by PHASTER $^{84}$ and masked from the respective alignments. Core genome alignments were used to construct maximum-likelihood (ML) trees using RaXML v8.2.12 with the generalized time-reversible (GTR) model and gamma distribution ${ }^{85}$. One hundred bootstrap replicates were performed to assess support for the phylogeny. ClonalFrameML v1.12 $2^{86}$ was used to account for recombination events and to correct branch lengths with the best RAxML tree as starting tree. To identify lineages enriched with invasive UPEC isolates, the pre-computed phylogeny of all 907 isolates or of CC69, CC73, or CC95 isolates was partitioned into clusters on multiple levels of resolution using Bayesian hierarchical clustering implemented in the R package fastbaps ${ }^{87}$. Clusters identified in different phylogenies were merged and the 129 clusters were tested for significant phenotype enrichment among UPEC isolates using Fisher's exact test. papGII+ lineages were defined based on patristic distances (cutoff 0.00032) in the maximum-likelihood tree of papGII+ isolates using RAMI ${ }^{88}$. A lower cutoff distance $(0.000052)$ was chosen for isolates of CC73 to address the heterogenous population structure within this CC and nested papGII+ clades combined. Phylogenetic trees and isolate metadata were visualized in $\mathrm{TTOL}^{89}$ and annotated using Inkscape 0.92. 
Genome-wide association studies. Clusters of orthologous genes (COGs) representing homologous genes with shared sequences were identified using Roary v3.12.0 with a BLASTp identity cutoff of $95 \%{ }^{90}$. The pan-genome of all isolates comprised 37,717 COGs, of which 3067 COGs represented the core genome (present in $\geq 99 \%$ of all isolates). Pan-genome-wide association studies (panGWAS) on the COG presence-absence matrix were performed on 30705 COGs (of which 2237 were present in all isolates) identified in 722 UPEC isolates using the $\mathrm{R}$ package treeWAS v1.0 with Bonferroni correction ${ }^{91}$. Fecal isolates were excluded from pan-GWAS and DBGWAS analyses due to their unknown urinary phenotype. Associations of each COG with invasive or non-invasive UPEC isolates were calculated using the Fisher's exact tests. The significance threshold (raw $P=1.42 \times$ $10^{-18}$, corresponding to a Bonferroni-corrected $P=4.04 \times 10^{-14}$ ) was determined from a simulated dataset accounting for population structure as described in treeWAS. Manhattan plots were generated using the R package CMplot (https:// github.com/YinLiLin/R-CMplot). PAIs in the genome of reference strain UMN026 were identified using Islander implemented in IslandViewer $4^{92}$. Genetic associations were additionally analyzed with DBGWAS v0.5.426 with a $q$-value threshold of 0.05 .

Genotyping. Phylogroups were determined according to phylogenetic clustering and supported by in silico typing using ClermonTyping v1. $4^{93}$. Multi-locus sequence types (ST) of the Achtmann scheme were determined using srst2 v0.2.094 with default settings or mlst v2.16.1 (https://github.com/tseemann/mlst). Identified STs were grouped into clonal complexes (CC) according to the scheme available at EnteroBase ${ }^{75}$. Alleles of type 1 fimbrial tip adhesin gene fim $H$ were identified using FimTyper v1.1 ${ }^{95}$. $\mathrm{H}$ and $\mathrm{O}$ serotypes were predicted in silico with the $\mathrm{EcOH}$ database $^{96}$ using srst2 v0.2.0 $0^{94}$ with default settings, or, in case no raw read data were available, ABRicate v0.9.3 (minimum sequence coverage/identity 70/90\%). Pointfinder ${ }^{97}$ was used to search for mutations in the quinolone resistancedetermining regions (QRDR). Resistance genes were identified using ABRicate v0.9.3 in conjunction with the resfinder database ${ }^{98}$ (minimum sequence coverage/ identity 70/90\%). Prophage genes and regions were identified using PHASTER ${ }^{84}$ with annotated genbank files as input. Clades of CC131 were confirmed using ABRicate v0.9.3 by screening assemblies against the clade B-specific allele of $p r f C$ identified in isolate KTE6 (minimum sequence coverage/identity 100/99\%), the clade C2-specific allele of $y b b W$ identified in isolate JJ1886 (minimum sequence coverage/identity 100/99\%), and the clade C1-specific allele of a gene with locus tag U12A_RS05235 identified in isolate U12A (exact matches).

Screening for virulence-associated genes. An E. coli virulence-associated gene database $\left(\mathrm{EcVGDB}^{99}\right)$ was compiled from two separate collections of $E$. coli virulence factors, ecoli_VF_collection ${ }^{100}$ and ecoli_vf (https://github.com/phacnml/ecoli_vf), which are both based on the virulence factor database VFDB ${ }^{101}$. Redundant sequences identified using $\mathrm{cdhit}^{102}$ were removed. The database was supplemented with additional virulence-associated genes (VAGs) from the literature, resulting in 1368 sequences representing 1072 VAGs of 14 virulence factor categories. Genome assemblies were screened against the EcVGDB using ABRicate (minimum sequence coverage/identity 70/90\%). The presence of alleles of the virulence gene families $p a p G$ and $a f a / d r a$ was additionally assessed by read mapping using srst $2^{94}$ using default settings, accepting hits with no or minor mismatches (SNPs or indels). iuc locus variants were determined by mapping reads to the reference genes shiF, shiFp, iutA1, and iutA2 obtained from the genomes of isolates CFT073, US06, and IAI39 (srst2 ${ }^{94}$, default settings). Hits with indels or holes were rejected. When read data was not available, iuc variants were determined using ABRicate with the three complete operons as reference (minimum sequence coverage/identity $70 / 90 \%$ ).

Identification and clustering of mobile genetic elements. Completely resolved papGII-containing PAIs, and PAIs or plasmids containing the iuc locus, were extracted from high-quality genome assemblies. To estimate their similarity, mash distance matrices were produced using mashtree v1.12 ${ }^{103}$, and the genetic elements hierarchically clustered in $\mathrm{R} v$ 33.5.3. papGII-containing PAIs were clustered by applying a mash distance cutoff of 0.04 . Mash distances are an estimate of sequence similarity calculated from the fraction of shared k-mers (Jaccard index) in MinHash sketches ${ }^{104}$. PyANI v0.2.9105 with BLAST $+{ }^{106}$ was used to identify the pairwise alignment coverage of PAIs. For the comparison of resolved PAIs of the same type across clonal complexes, the lower of the two alignment coverage values resulting from subject and query choice of each pair was chosen. To predict papGII+ PAI types in papGII+ isolates with insufficient sequence assemblies, their sequencing reads were mapped to the 42 resolved papGII+ PAIs using srst $2^{94}$ with default settings. Identified hits with $\geq 90 \%$ identity and $\geq 90 \%$ coverage were screened for homologs with no or minor mismatches (SNPs or indels) and assigned to the corresponding papGII+ PAI type. Hits with large deletions or truncations ("holes") were counted as mismatches. Hits in isolates with both papGII+ and pap GIII-containing PAIs, particularly observed in papGII+ lineages of CC73, could lead to ambiguous hits which were excluded due to possible recombination events between the two pap-containing PAIs.
Detection of integration sites of papGII-containing PAls. ABRicate v0.9.3 (minimum sequence coverage/identity 70/90\%) was used to query the genomic position of papGII and housekeeping genes surrounding the six identified integration sites (tRNA-pheV: speC - kpsFII or gspM/yghD; tRNA-pheU: yjdC - cadC; tRNA-selC: yicL - yicj; tRNA-leuX: yjgB - gntP/uxuA; ula: ulaE - ulaD; gln: glnP $g \ln H)$ in each genome assembly. Integration sites were then inferred from the chromosomal distance between papGII and the surrounding housekeeping genes. The integration site could not be inferred from assemblies with highly fragmented PAIs, which was often the case in isolates with multiple pap operons.

Multiple sequence alignments and visualization of genomes and PAls. Gene and protein sequences were aligned using Muscle v3.8.31 107 and their phylogenetic relationship reconstructed using a GTR model in Mega-X v10.0.5 $5^{108}$. Percent sequence identity matrices were calculated using Clustal2.1 ${ }^{109}$. Schematic representations and comparisons of genomes and PAIs were generated using EasyFig 2.2.3110.

Statistical tests. Statistical analyses were performed using R v3.5.3. Frequency counts were compared using two-tailed Fisher's exact test, while continuous variables were analyzed using two-tailed Mann-Whitney U test. Bonferroni adjusted $P$ values of $<0.05$ were considered to reflect statistical significance, except in the GWAS analyses.

Material availability. Requests for obtaining clinical isolates collected as part of this study should be addressed to the corresponding author. Exchange of clinical isolates should always be in agreement with the University of Antwerp.

Ethics. Ethical approval for the study was received from the ethics committee UZA (approval No. 17/08/081, No. 20/11/119, and No. 18/10/122). Written informed consent was obtained from all subjects participating in the clinical investigation or, when the participant was not capable of giving consent, by his or her legal representative.

Reporting summary. Further information on research design is available in the Nature Research Reporting Summary linked to this article.

\section{Data availability}

Illumina and PacBio reads generated for this study are available at the NCBI Sequence Read Archive (SRA) under BioProject no. PRJNA592372. Complete or draft genome assemblies have been submitted to NCBI GenBank. Individual accession numbers are provided in Supplementary Data 1. Sequences of papGII+ PAIs and the curated E. coli virulence gene database (EcVGDB) are provided at https://github.com/MBiggel/ UPEC_study (https://doi.org/10.5281/zenodo.4079473). An interactive version of the core genome phylogeny of the $907 \mathrm{E}$. coli isolates is accessible at https://microreact.org/ project/O4QAYAJWw. All other relevant data are available from the corresponding authors. Public data utilized in this study include genomic data (accession numbers provided in Supplementary Data 1 and 10), the databases plasmidfinder, resfinder, EcOH, ecoli_vf, ecoli_VF_collection, and the EnteroBase ST/CC scheme.

Received: 6 April 2020; Accepted: 27 October 2020; Published online: 24 November 2020

\section{References}

1. Vihta, K.-D. et al. Trends over time in Escherichia coli bloodstream infections, urinary tract infections, and antibiotic susceptibilities in Oxfordshire, UK, 1998-2016: a study of electronic health records. Lancet Infect. Dis. 18, 1138-1149 (2018).

2. Abernethy, J. et al. Epidemiology of Escherichia coli bacteraemia in England results of an enhanced sentinel surveillance programme. J. Hosp. Infect. 95, 365-375 (2017).

3. van Hout, D. et al. Extended-spectrum beta-lactamase (ESBL)-producing and non-ESBL-producing Escherichia coli isolates causing bacteremia in the Netherlands (2014-2016) differ in clonal distribution, antimicrobial resistance gene and virulence gene content. PLoS ONE 15, e0227604 (2020).

4. Johnson, J. R. \& Russo, T. A. Extraintestinal pathogenic Escherichia coli: 'The other bad E coli. J. Lab. Clin. Med. 139, 155-162 (2002).

5. Klein, R. D. \& Hultgren, S. J. Urinary tract infections: microbial pathogenesis, host-pathogen interactions and new treatment strategies. Nat. Rev. Microbiol. https://doi.org/10.1038/s41579-020-0324-0 (2020).

6. Robins-Browne, R. M. et al. Are Escherichia coli pathotypes still relevant in the era of whole-genome sequencing? Front. Cell. Infect. Microbiol. 6, 1-9 (2016).

7. Köhler, C.-D. \& Dobrindt, U. What defines extraintestinal pathogenic Escherichia coli. Int. J. Med. Microbiol. 301, 642-647 (2011). 
8. Schreiber, H. L. IV, Spaulding, C. N., Dodson, K. W., Livny, J. \& Hultgren, S. J. One size doesn't fit all: unraveling the diversity of factors and interactions that drive E. coli urovirulence. Ann. Transl. Med. 5, 28-28 (2017).

9. Ingle, D. J. et al. Evolution of atypical enteropathogenic E. coli by repeated acquisition of LEE pathogenicity island variants. Nat. Microbiol. 1, 15010 (2016).

10. von Mentzer, A. et al. Identification of enterotoxigenic Escherichia coli (ETEC) clades with long-term global distribution. Nat. Genet. 46, 1321-1326 (2014).

11. Riley, L. W. Pandemic lineages of extraintestinal pathogenic Escherichia coli. Clin. Microbiol. Infect. 20, 380-390 (2014).

12. Kallonen, T. et al. Systematic longitudinal survey of invasive Escherichia coli in England demonstrates a stable population structure only transiently disturbed by the emergence of ST131. Genome Res. 27, 1437-1449 (2017).

13. Fibke, C. D. et al. Genomic epidemiology of major extraintestinal pathogenic Escherichia coli lineages causing urinary tract infections in young women across Canada. Open Forum Infect. Dis. 6, 1-11 (2019).

14. Ben Zakour, N. L. et al. Sequential acquisition of virulence and fluoroquinolone resistance has shaped the evolution of Escherichia coli ST131. MBio 7, 1-11 (2016).

15. Salipante, S. J. et al. Large-scale genomic sequencing of extraintestinal pathogenic Escherichia coli strains. Genome Res. 25, 119-128 (2015).

16. Galardini, M. et al. Major role of the high-pathogenicity island (HPI) in the intrinsic extra-intestinal virulence of Escherichia coli revealed by a genomewide association study. bioRxiv https://doi.org/10.1101/712034 (2019).

17. Goswami, C. et al. Genetic analysis of invasive Escherichia coli in Scotland reveals determinants of healthcare-associated versus community-acquired infections. Microb. Genomics 4, 1-15 (2018).

18. Nielsen, K. L. et al. Whole-genome comparison of urinary pathogenic Escherichia coli and faecal isolates of UTI patients and healthy controls. Int. J. Med. Microbiol. 307, 497-507 (2017).

19. Spurbeck, R. R. et al. Fimbrial profiles predict virulence of uropathogenic Escherichia coli strains: contribution of Ygi and Yad fimbriae. Infect. Immun. 79, 4753-4763 (2011).

20. Marrs, C. F. et al. Variations in 10 putative uropathogen virulence genes among urinary, faecal and peri-urethral Escherichia coli. J. Med. Microbiol. 51, 138-142 (2002).

21. Johnson, J. R., Owens, K., Gajewski, A. \& Kuskowski, M. A. Bacterial characteristics in relation to clinical source of Escherichia coli Isolates from women with acute cystitis or pyelonephritis and uninfected women. J. Clin. Microbiol. 43, 6064-6072 (2005).

22. Johnson, J. R. papG alleles among Escherichia coli strains causing urosepsis: associations with other bacterial characteristics and host compromise. Infect. Immun. 66, 4568-4571 (1998).

23. Ambite, I. et al. Fimbriae reprogram host gene expression-divergent effects of P and type 1 fimbriae. PLOS Pathog. 15, e1007671 (2019).

24. Welch, R. A. et al. Extensive mosaic structure revealed by the complete genome sequence of uropathogenic Escherichia coli. Proc. Natl Acad. Sci. USA 99, 17020-17024 (2002).

25. Schmidt, H. \& Hensel, M. Pathogenicity islands in bacterial pathogenesis. Clin. Microbiol. Rev. 17, 14-56 (2004).

26. Jaillard, M. et al. A fast and agnostic method for bacterial genome-wide association studies: Bridging the gap between k-mers and genetic events. PLOS Genet. 14, e1007758 (2018).

27. Johnson, J. R., O’Bryan, T. T., Kuskowski, M. \& Maslow, J. N. Ongoing horizontal and vertical transmission of virulence genes and papA alleles among Escherichia coli blood isolates from patients with diverse-source bacteremia. Infect. Immun. 69, 5363-5374 (2001).

28. Jantausch, B. A. \& Hull, S. I. Restriction fragment length polymorphism of PCR amplified papE gene products is correlated with complete serotype among uropathogenic Escherichia coli isolates. Microb. Pathog. 20, 351-360 (1996).

29. Dobrindt, U., Hochhut, B., Hentschel, U. \& Hacker, J. Genomic islands in pathogenic and environmental microorganisms. Nat. Rev. Microbiol. 2, 414-424 (2004).

30. Montero, D. A. et al. Locus of Adhesion and Autoaggregation (LAA), a pathogenicity island present in emerging Shiga Toxin-producing Escherichia coli strains. Sci. Rep. 7, 1-13 (2017).

31. Jouve, M. et al. Adhesion to and invasion of HeLa cells by pathogenic Escherichia coli carrying the afa-3 gene cluster are mediated by the AfaE and AfaD proteins, respectively. Infect. Immun. 65, 4082-4089 (1997).

32. Le Bouguenec, C. et al. Characterization of AfaE adhesins produced by extraintestinal and intestinal human Escherichia coli isolates: PCR assays for detection of afa adhesins that do or do not recognize Dr blood group antigens. J. Clin. Microbiol. 39, 1738-1745 (2001).

33. Servin, A. L. Pathogenesis of human diffusely adhering Escherichia coli expressing Afa/Dr adhesins (Afa/Dr DAEC): current insights and future challenges. Clin. Microbiol. Rev. 27, 823-869 (2014).
34. Ulett, G. C. et al. Functional analysis of antigen 43 in uropathogenic Escherichia coli reveals a role in long-term persistence in the urinary tract. Infect. Immun. 75, 3233-3244 (2007).

35. Lloyd, A. L., Smith, S. N., Eaton, K. A. \& Mobley, H. L. T. Uropathogenic Escherichia coli suppresses the host inflammatory response via pathogenicity island genes sisA and sisB. Infect. Immun. 77, 5322-5333 (2009).

36. Garcia, E. C., Brumbaugh, A. R. \& Mobley, H. L. T. Redundancy and specificity of Escherichia coli iron acquisition systems during urinary tract infection. Infect. Immun. 79, 1225-1235 (2011).

37. Robinson, A. E., Heffernan, J. R. \& Henderson, J. P. The iron hand of uropathogenic Escherichia coli: the role of transition metal control in virulence. Future Microbiol. 13, 813-829 (2018).

38. Wurpel, D. J., Moriel, D. G., Totsika, M., Easton, D. M. \& Schembri, M. A. Comparative analysis of the uropathogenic Escherichia coli surface proteome by tandem mass-spectrometry of artificially induced outer membrane vesicles. J. Proteom. 115, 93-106 (2015).

39. Di Lorenzo, M. \& Stork, M. in Plasmids: Biology and Impact in Biotechnology and Discovery Vol. 2, 577-597 (American Society of Microbiology, 2015).

40. Johnson, J. R. et al. Host characteristics and bacterial traits predict experimental virulence for Escherichia coli bloodstream isolates from patients with urosepsis. Open Forum Infect. Dis. 2, ofu121 (2015).

41. Johnson, J. R. \& Stell, A. L. Extended virulence genotypes of Escherichia coli strains from patients with urosepsis in relation to phylogeny and host compromise. J. Infect. Dis. 181, 261-272 (2000).

42. Spurbeck, R. R. et al. Escherichia coli isolates that carry vat, fyuA, chuA, and $y f c V$ efficiently colonize the urinary tract. Infect. Immun. 80, 4115-4122 (2012).

43. Schreiber, H. L. et al. Bacterial virulence phenotypes of Escherichia coli and host susceptibility determine risk for urinary tract infections. Sci. Transl. Med. 9, eaaf1283 (2017).

44. Lam, M. M. C. et al. Tracking key virulence loci encoding aerobactin and salmochelin siderophore synthesis in Klebsiella pneumoniae. Genome Med. 10, 77 (2018).

45. Gordon, D. M. et al. Fine-scale structure analysis shows epidemic patterns of clonal complex 95, a cosmopolitan Escherichia coli. mSphere 2, 1-15 (2017).

46. Petty, N. K. et al. Global dissemination of a multidrug resistant Escherichia coli clone. Proc. Natl Acad. Sci. USA 111, 5694-5699 (2014).

47. Johanson, I.-M., Plos, K., Marklund, B.-I. \& Svanborg, C. Pap, papG and prsG DNA sequences in Escherichia coli from the fecal flora and the urinary tract. Microb. Pathog. 15, 121-129 (1993).

48. Roberts, J. A. et al. The Gal(alpha 1-4)Gal-specific tip adhesin of Escherichio coli P-fimbriae is needed for pyelonephritis to occur in the normal urinary tract. Proc. Natl Acad. Sci USA. 91, 11889-11893 (1994).

49. Hagberg, L. et al. Contribution of adhesion to bacterial persistence in the mouse urinary tract. Infect. Immun. 40, 265-272 (1983).

50. Tseng, C. C. et al. PapG II adhesin in the establishment and persistence of Escherichia coli infection in mouse kidneys. Kidney Int. 71, 764-770 (2007).

51. Tenaillon, O., Skurnik, D., Picard, B. \& Denamur, E. The population genetics of commensal Escherichia coli. Nat. Rev. Microbiol. 8, 207-217 (2010).

52. Johnson, J. R. Virulence factors in Escherichia coli urinary tract infection. Clin. Microbiol. Rev. 4, 80-128 (1991).

53. Biggel, M. et al. Asymptomatic bacteriuria in older adults: the most fragile women are prone to long-term colonization. BMC Geriatr. 19, 170 (2019).

54. Ragnarsdóttir, B., Lutay, N., Grönberg-Hernandez, J., Köves, B. \& Svanborg C. Genetics of innate immunity and UTI susceptibility. Nat. Rev. Urol. 8, 449-468 (2011).

55. Kanamori, H. et al. Genomic analysis of multidrug-resistant Escherichia coli from North Carolina Community Hospitals: ongoing circulation of CTX-MProducing ST131-H30Rx and ST131-H30R1 strains. Antimicrob. Agents Chemother. 61, 1-13 (2017).

56. Bonat, G. et al. European association of urology guidelines on urological infections. EAU Guidel. 1-66 (2018).

57. Nowrouzian, F. L., Wold, A. E. \& Adlerberth, I. Escherichia coli strains belonging to phylogenetic group B2 have superior capacity to persist in the intestinal microflora of infants. J. Infect. Dis. 191, 1078-1083 (2005).

58. Tullus, K., Kühn, I., Ørskov, I., Ørskov, F. \& Möllby, R. The importance of P and type 1 fimbriae for the persistence of Escherichia coli in the human gut. Epidemiol. Infect. 108, 415-421 (1992).

59. Vandemaele, F. J., Mugasa, J. P., Vandekerchove, D. \& Goddeeris, B. M. Predominance of the papGII allele with high sequence homology to that of human isolates among avian pathogenic Escherichia coli (APEC). Vet. Microbiol. 97, 245-257 (2003).

60. Johnson, T. J. et al. Comparison of extraintestinal pathogenic Escherichia coli strains from human and avian sources reveals a mixed subset representing potential zoonotic pathogens. Appl. Environ. Microbiol. 74, 7043-7050 (2008)

61. Ewers, C., Antao, E.-M., Diehl, I., Philipp, H.-C. \& Wieler, L. H. Intestine and environment of the chicken as reservoirs for extraintestinal pathogenic 
Escherichia coli strains with zoonotic potential. Appl. Environ. Microbiol. 75, 184-192 (2009).

62. Manges, A. R. \& Johnson, J. R. Reservoirs of extraintestinal pathogenic Escherichia coli. Microbiol. Spectr. 3, 1-12 (2015).

63. Magruder, M. et al. Gut uropathogen abundance is a risk factor for development of bacteriuria and urinary tract infection. Nat. Commun. 10, 5521 (2019).

64. Thänert, R. et al. Comparative genomics of antibiotic-resistant uropathogens implicates three routes for recurrence of urinary tract infections. MBio 10, 1-16 (2019).

65. Khandige, S., Kronborg, T., Uhlin, B. E. \& Møller-Jensen, J. sRNA-mediated regulation of P-fimbriae phase variation in uropathogenic Escherichia coli. PLoS Pathog. 11, 1-23 (2015).

66. Blomfield, I. C. The regulation of pap and type 1 fimbriation in Escherichia coli. Adv. Microb. Physiol. 45, 1-49 (2001).

67. Nielsen, K. L., Dynesen, P., Larsen, P. \& Frimodt-Møller, N. Faecal Escherichia coli from patients with $E$. coli urinary tract infection and healthy controls who have never had a urinary tract infection. J. Med. Microbiol. 63, 582-589 (2014).

68. Kaukonen, K.-M., Bailey, M., Pilcher, D., Cooper, D. J. \& Bellomo, R. Systemic inflammatory response syndrome criteria in defining severe sepsis. N. Engl. J. Med. 372, 1629-1638 (2015).

69. Drekonja, D. M., Kuskowski, M. A., Anway, R., Johnston, B. D. \& Johnson, J. R. The niche for Escherichia coli sequence type 131 among veterans: urinary tract abnormalities and long-term care facilities. Open Forum Infect. Dis. 3, 1-6 (2016).

70. Sannes, M. R., Kuskowski, M. A. \& Johnson, J. R. Antimicrobial resistance of Escherichia coli strains isolated from urine of women with cystitis or pyelonephritis and feces of dogs and healthy humans. J. Am. Vet. Med. Assoc. 225, 368-373 (2004).

71. Talan, D. A. et al. Comparison of ciprofloxacin (7 days) and trimethoprimsulfamethoxazole (14 days) for acute uncomplicated pyelonephritis in women. JAMA 283, 1583 (2000).

72. Johnson, J. R. et al. Accessory traits and phylogenetic background predict Escherichia coli extraintestinal virulence better than does ecological source. J. Infect. Dis. 219, 121-132 (2018).

73. Birgy, A. et al. Diversity and trends in population structure of ESBL-producing Enterobacteriaceae in febrile urinary tract infections in children in France from 2014 to 2017. J. Antimicrob. Chemother. 96-105, https://doi.org/10.1093/ $\mathrm{jac} / \mathrm{dkz} 423$ (2019).

74. Syre, H. et al. Microbial risk factors for treatment failure of pivmecillinam in community-acquired urinary tract infections caused by ESBL-producing Escherichia coli. APMIS apm.13013, https://doi.org/10.1111/apm.13013 (2020).

75. Zhou, Z., Alikhan, N., Mohamed, K., Fan, Y. \& Achtman, M. The EnteroBase user's guide, with case studies on Salmonella transmissions, Yersinia pestis phylogeny, and Escherichia core genomic diversity. Genome Res. 30, 138-152 (2020).

76. Bankevich, A. et al. SPAdes: a new genome assembly algorithm and its applications to single-cell sequencing. J. Comput. Biol. 19, 455-477 (2012).

77. Chin, C.-S. et al. Nonhybrid, finished microbial genome assemblies from longread SMRT sequencing data. Nat. Methods 10, 563-569 (2013).

78. Wick, R. R., Judd, L. M., Gorrie, C. L. \& Holt, K. E. Unicycler: resolving bacterial genome assemblies from short and long sequencing reads. PLOS Comput. Biol. 13, e1005595 (2017).

79. Arredondo-Alonso, S. et al. Mlplasmids: a user-friendly tool to predict plasmid- and chromosome-derived sequences for single species. Microb. Genomics 4, e000224 (2018).

80. Carattoli, A. et al. In silico detection and typing of plasmids using PlasmidFinder and plasmid multilocus sequence typing. Antimicrob. Agents Chemother. 58, 3895-3903 (2014).

81. Gurevich, A., Saveliev, V., Vyahhi, N. \& Tesler, G. QUAST: quality assessment tool for genome assemblies. Bioinformatics 29, 1072-1075 (2013).

82. Seemann, T. Prokka: rapid prokaryotic genome annotation. Bioinformatics 30, 2068-2069 (2014).

83. Treangen, T. J., Ondov, B. D., Koren, S. \& Phillippy, A. M. The Harvest suite for rapid core-genome alignment and visualization of thousands of intraspecific microbial genomes. Genome Biol. 15, 524 (2014).

84. Arndt, D. et al. PHASTER: a better, faster version of the PHAST phage search tool. Nucleic Acids Res. 44, W16-W21 (2016).

85. Stamatakis, A. RAxML version 8: a tool for phylogenetic analysis and postanalysis of large phylogenies. Bioinformatics 30, 1312-1313 (2014).

86. Didelot, X. \& Wilson, D. J. ClonalFrameML: efficient inference of recombination in whole bacterial genomes. PLoS Comput. Biol. 11, 1-18 (2015).

87. Tonkin-Hill, G., Lees, J. A., Bentley, S. D., Frost, S. D. W. \& Corander, J. Fast hierarchical Bayesian analysis of population structure. Nucleic Acids Res. 47, 5539-5549 (2019).
88. Pommier, T., Canbäck, B., Lundberg, P., Hagström, Å. \& Tunlid, A. RAMI: a tool for identification and characterization of phylogenetic clusters in microbial communities. Bioinformatics 25, 736-742 (2009).

89. Letunic, I. \& Bork, P. Interactive Tree Of Life (iTOL) v4: recent updates and new developments. Nucleic Acids Res. 47, W256-W259 (2019).

90. Page, A. J. et al. Roary: rapid large-scale prokaryote pan genome analysis. Bioinformatics 31, 3691-3693 (2015).

91. Collins, C. \& Didelot, X. A phylogenetic method to perform genome-wide association studies in microbes that accounts for population structure and recombination. PLoS Comput. Biol. 14, 1-21 (2018).

92. Bertelli, C. et al. IslandViewer 4: expanded prediction of genomic islands for larger-scale datasets. Nucleic Acids Res. 45, W30-W35 (2017).

93. Beghain, J. et al. ClermonTyping: an easy-to-use and accurate in silico method for Escherichia genus strain phylotyping. Microb. Genomics 4, 1-8 (2018).

94. Inouye, M. et al. SRST2: Rapid genomic surveillance for public health and hospital microbiology labs. Genome Med. 6, 90 (2014).

95. Roer, L. et al. Development of a web tool for Escherichia coli subtyping based on fimH alleles. J. Clin. Microbiol. 55, 2538-2543 (2017).

96. Ingle, D. J. et al. In silico serotyping of $E$. coli from short read data identifies limited novel O-loci but extensive diversity of O:H serotype combinations within and between pathogenic lineages. Microb. Genomics 2, e000109 (2016)

97. Zankari, E. et al. PointFinder: a novel web tool for WGS-based detection of antimicrobial resistance associated with chromosomal point mutations in bacterial pathogens. J. Antimicrob. Chemother. 72, 2764-2768 (2017).

98. Zankari, E. et al. Identification of acquired antimicrobial resistance genes. J. Antimicrob. Chemother. 67, 2640-2644 (2012).

99. Biggel, M. EcVGDB. Zenodo https://doi.org/10.5281/zenodo.4079473 (2020).

100. Leimbach, A. ecoli_VF_collection: v0.1. Zenodo https://doi.org/10.5281/ zenodo.56686 (2016).

101. Chen, L., Zheng, D., Liu, B., Yang, J. \& Jin, Q. VFDB 2016: hierarchical and refined dataset for big data analysis-10 years on. Nucleic Acids Res. 44, D694-D697 (2016).

102. Li, W. \& Godzik, A. Cd-hit: a fast program for clustering and comparing large sets of protein or nucleotide sequences. Bioinformatics 22, 1658-1659 (2006).

103. Katz, L. et al. Mashtree: a rapid comparison of whole genome sequence files. J. Open Source Softw. 4, 1762 (2019).

104. Ondov, B. D. et al. Mash: fast genome and metagenome distance estimation using MinHash. Genome Biol. 17, 132 (2016).

105. Pritchard, L., Glover, R. H., Humphris, S., Elphinstone, J. G. \& Toth, I. K. Genomics and taxonomy in diagnostics for food security: soft-rotting enterobacterial plant pathogens. Anal. Methods 8, 12-24 (2016).

106. Camacho, C. et al. BLAST+: architecture and applications. BMC Bioinforma. 10, 421 (2009).

107. Edgar, R. C. MUSCLE: a multiple sequence alignment method with reduced time and space complexity. BMC Bioinforma. 5, 1-19 (2004).

108. Kumar, S., Stecher, G., Li, M., Knyaz, C. \& Tamura, K. MEGA X: molecular evolutionary genetics analysis across computing platforms. Mol. Biol. Evol. 35, $1547-1549$ (2018)

109. Madeira, F. et al. The EMBL-EBI search and sequence analysis tools APIs in 2019. Nucleic Acids Res. 47, W636-W641 (2019).

110. Sullivan, M. J., Petty, N. K. \& Beatson, S. A. Easyfig: a genome comparison visualizer. Bioinformatics 27, 1009-1010 (2011)

111. Jolley, K. A. \& Maiden, M. C. J. BIGSdb: Scalable analysis of bacterial genome variation at the population level. BMC Bioinforma. 11, 595 (2010).

112. Stork, C. et al. Characterization of asymptomatic bacteriuria Escherichia coli isolates in search of alternative strains for efficient bacterial interference against uropathogens. Front. Microbiol. 9, 1-18 (2018).

113. Coussement, J. et al. Host and microbial factors in kidney transplant recipients with Escherichia coli acute pyelonephritis or asymptomatic bacteriuria: a prospective study using whole-genome sequencing. Nephrol. Dial. Transpl. 34, 878-885 (2019).

114. Skjøt-Rasmussen, L. et al. Persisting clones of Escherichia coli isolates from recurrent urinary tract infection in men and women. J. Med. Microbiol 60, 550-554 (2011).

115. Ejrnæs, K. et al. Characteristics of Escherichia coli causing persistence or relapse of urinary tract infections: Phylogenetic groups, virulence factors and biofilm formation. Virulence 2, 528-537 (2011).

116. Czaja, C. A. C. et al. Prospective cohort study of microbial and inflammatory events immediately preceding Escherichia coli recurrent urinary tract infection in women. J. Infect. Dis. 200, 528-536 (2009).

117. Sannes, M. R., Kuskowski, M. A. \& Johnson, J. R. Geographical distribution of antimicrobial resistance among Escherichia coli causing acute uncomplicated pyelonephritis in the United States. FEMS Immunol. Med. Microbiol. 42, 213-218 (2004).

118. Skiøt-Rasmussen, L., Ejrnæs, K., Lundgren, B., Hammerum, A. M. \& FrimodtMøller, N. Virulence factors and phylogenetic grouping of Escherichia coli isolates from patients with bacteraemia of urinary tract origin relate to sex and hospitalvs. community-acquired origin. Int. J. Med. Microbiol. 302, 129-134 (2012). 
119. Dale, A. P. et al. Genomes of Escherichia coli bacteraemia isolates originating from urinary tract foci contain more virulence-associated genes than those from non-urinary foci and neutropaenic hosts. J. Infect. 77, 534-543 (2018).

120. Sannes, M. R., Kuskowski, M. A., Owens, K., Gajewski, A. \& Johnson, J. R. Virulence factor profiles and phylogenetic background of Escherichia coli isolates from veterans with bacteremia and uninfected control subjects. J. Infect. Dis. 190, 2121-2128 (2004).

\section{Acknowledgements}

We want to thank all participants who donated samples for this study; the staff of the long-term care facilities OCMW Destelbergen and WZC Immaculata Edegem, in particular Marleen Paelinck, Wouter Rogiest, and Hans Van Braeckel; Stefan Heytens and Katrien Latour for help in organizing the sample collections and reviewing patient data; staff of the Laboratory of Medical Microbiology (LMM) for assisting in genome sequencing; Julien Coussement and Brian Johnston for sharing bacterial isolates or sequencing data; and all scientists who deposited their sequencing data on publicly available archives, which has made this study possible. This publication made use of the PubMLST website (http://pubmlst.org/) developed by Keith Jolley ${ }^{111}$ and sited at the University of Oxford. The development of that website was funded by the Wellcome Trust. The computational resources and services used in this work were provided by the VSC (Flemish Supercomputer Center), funded by the Research Foundation-Flanders (FWO) and the Flemish Government-department EWI. The work has received funding from the European Union's Horizon 2020 research and innovation program under the Marie Skłodowska-Curie grant agreement No. 675412. This work is also supported in part by Office of Research and Development, Department of Veterans Affairs (U.S.).

\section{Author contributions}

M.B., P.M., and S.V.P. designed the study. M.B. collected the samples and performed the experimental analyses. V.M. and J.J. contributed to isolate collections. M.B. performed the bioinformatics analyses with input from S.V.P. and P.M. M.B, S.V.P., and P.M. wrote the manuscript. This was revised by B.X., H.G., J.J., K.N., and N.F.-M. All authors have read and approved the manuscript.

\section{Competing interests}

The authors declare no competing interests.

\section{Additional information}

Supplementary information is available for this paper at https://doi.org/10.1038/s41467020-19714-9.

Correspondence and requests for materials should be addressed to M.B. or S.V.P.

Peer review information Nature Communications thanks Astrid von Mentzer and the other, anonymous, reviewer(s) for their contribution to the peer review of this work. Peer reviewer reports are available.

Reprints and permission information is available at http://www.nature.com/reprints

Publisher's note Springer Nature remains neutral with regard to jurisdictional claims in published maps and institutional affiliations.

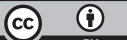

Open Access This article is licensed under a Creative Commons Attribution 4.0 International License, which permits use, sharing, adaptation, distribution and reproduction in any medium or format, as long as you give appropriate credit to the original author(s) and the source, provide a link to the Creative Commons license, and indicate if changes were made. The images or other third party material in this article are included in the article's Creative Commons license, unless indicated otherwise in a credit line to the material. If material is not included in the article's Creative Commons license and your intended use is not permitted by statutory regulation or exceeds the permitted use, you will need to obtain permission directly from the copyright holder. To view a copy of this license, visit http://creativecommons.org/ licenses/by/4.0/.

(C) Crown 2020 